\title{
Mineral Fertilizer Use for Optimal Groundnut Production in the Sudano-Guinean and Sudanian Zones of Benin
}

\author{
Faki O. Chabi'" , Gustave D. Dagbenonbakin ${ }^{2}$, Emile C. Agbangba ${ }^{3}$, Issa Mouftaou ${ }^{1}$, \\ Augustin 0. Bankolé1, Brice T. Oussou ${ }^{4}$, Léonard E. Ahoton'1, Guillaume L. Amadji ${ }^{5}$, Aliou Saïdou ${ }^{1}$ \\ ${ }^{1}$ Integrated Soil and Crop Management Research Unit, Laboratory of Soil Sciences, Crop Sciences School, Faculty of Agronomic \\ Sciences, University of Abomey-Calavi, Cotonou, Benin \\ ${ }^{2}$ Institut National des Recherches Agricoles du Benin, Cotonou, Benin \\ ${ }^{3}$ Laboratory of Research in Applied Biology, Department of Environmental Engineering, Polytechnic School of Abomey-Calavi, \\ University of Abomey-Calavi, Cotonou, Benin \\ ${ }^{4}$ Laboratoire des Sciences du Sol, Eau et Environnement (LSSEE), Centre de Recherche d'Agonkanmey, Institut National des \\ Recherches Agricoles du Benin (INRAB), Cotonou, Benin \\ ${ }^{5}$ Laboratory of Soil Science, Faculty of Agronomic Sciences, University of Abomey-Calavi, Abomey-Calavi, Benin \\ Email: "chabifaki@gmail.com
}

How to cite this paper: Chabi, O.F., Dagbenonbakin, G.D., Agbangba, E.C., Mouftaou, I., Bankole, A.O., Oussou, B.T., Ahoton, L.E., Amadji, G.L. and Saidou A. (2021) Mineral Fertilizer Use for Optimal Groundnut Production in the Sudano-Guinean and Sudanian Zones of Benin. Open Journal of Soil Science, 11, 72-92. https://doi.org/10.4236/ojss.2021.112005

Received: January 8, 2021

Accepted: February 23, 2021

Published: February 26, 2021

Copyright $\odot 2021$ by author(s) and Scientific Research Publishing Inc. This work is licensed under the Creative Commons Attribution International License (CC BY 4.0).

http://creativecommons.org/licenses/by/4.0/ (c) (i) Open Access

\begin{abstract}
This study aims to determine the optimal $\mathrm{N}, \mathrm{P}, \mathrm{K}, \mathrm{Mg}$ and $\mathrm{Zn}$ rates for groundnut production on Ferric and Plintic Luvisol in the Sudano-Guinean and Sudanian zones of Benin Republic. Two years (2018 and 2019) experiment was carried out in the municipality of Ouessè in the Sudano-Guinean zone and Bembèrèkè in the Sudanian zone. The tested nutrient doses were $\mathrm{N}$ $\left(0,20\right.$ and $\left.40 \mathrm{~kg} \cdot \mathrm{ha}^{-1}\right), \mathrm{P}\left(0,25\right.$ and $\left.50 \mathrm{~kg} \cdot \mathrm{ha}^{-1}\right), \mathrm{K}\left(0,20\right.$ and $\left.40 \mathrm{~kg} \cdot \mathrm{ha}^{-1}\right), \mathrm{Mg}$ $\left(0,15\right.$ and $\left.30 \mathrm{~kg} \cdot \mathrm{ha}^{-1}\right)$ and $\mathrm{Zn}\left(0,4\right.$ and $\left.8 \mathrm{~kg} \cdot \mathrm{ha}^{-1}\right)$. The Box and Behnken rotating design is used to define the $\mathrm{N}, \mathrm{P}, \mathrm{K}, \mathrm{Mg}$ and $\mathrm{Zn}$ rate combinations leading to 46 combinations. A completely randomized bloc design was setting up considering farmers as replication. In total, four farmers' fields were selected. A one-way analysis of variance is carried out on yield data, using the linear mixed-effect model. Response surface analyses were used to determine the optimal doses for each $\mathrm{N}, \mathrm{P}, \mathrm{K}, \mathrm{Mg}$ and $\mathrm{Zn}$. Nodule production (6.5 times higher than the control), number of gynophores (2.8 times higher than the control) and root length $(19.2 \pm 0.2 \mathrm{~cm})$ of groundnut plants were significantly $(\mathrm{p}=0.0001)$ improved with nutrient application. The response surface analysis shows that treatments N-P-K-Mg-Zn of 16.01-20.18-6.70-5.65-2.47 (in the Sudano-Guinean zone) and 13.1-25.07-11.47-0-1.82 (in the Sudanian zone) are the optimal rates that have induced optimal yield of $2.1 \mathrm{t} \cdot \mathrm{ha}^{-1}$ (i.e.
\end{abstract}


2.5 times the yield in the farmers' field) pod yield and the best return on investment per hectare. Nevertheless, for a sustainable groundnut production, treatment 13.1-25.07-11.47-20-1.82 is suggested as regular $\mathrm{K}$ input is required for the respect of the fertilization laws.

\section{Keywords}

Soil Fertility, Response Surface, Box and Behnken Design, Modeling, Micronutrient

\section{Introduction}

Groundnut is one of the protein sources widely used for both human and animal nutrition in developing countries [1]. Leguminous crops represent high economic important crops in the traditional cropping system with a wide ecological adaptability [2]. Through the symbiotic association between legumes and rhizobium bacteria, atmospheric nitrogen is fixed in soil in mineral form [3]. Through this process, legumes improve soil nitrogen stock for the subsequent crops [4] [5]. In addition, some legumes, especially groundnut, contribute to the solubilization of insoluble phosphorus in the soils [6]. Legumes improve the physical environment and soil microbial activity as well as replenishment of soil organic matter stock [7].

Unfortunately in the traditional cropping system, groundnut is cultivated without fertilizer application, despite the low soil fertility level leading to crop yield decrease. For sustainable groundnut production, there is a need to develop appropriate fertilizer formula knowing that, as mentioned by [5], total amount of nitrogen fixed by a legume is not enough and cannot satisfy crop nitrogen requirement. Although nitrogen-based fertilization is regarded by several authors as a precursor of crop yield formation, not so important for groundnut yield [8] [9] when the soil fertility is at its appropriate level. Due to the oily nature of the seeds, the plants take up large amount of nutrients from the soil, leading to soil fertility depletion when nutrients are not replenished [10]. Furthermore, legumes with high harvest indices do not improve soil fertility, because a large amount of nutrients are in the seeds. But they rather deplete soil nitrogen [11]. It is reported that, soil nutrient deficiencies are the major cause of failure in legumes' root nodulation and affecting nitrogen fixation [10]. Thus, nutritional imbalance, mainly of macronutrients and micronutrients, contributed to yield losses with drawbacks on oilseed quality [12].

However, balanced mineral fertilization of groundnut considering both macronutrients and micronutrients would be a strategy to improve crop yield and soil fertility [13] [14]. Micronutrient application for leguminous crops plays an important role in improving crop yield. Due to intercropping practices, a single micronutrient deficiency can affect crop productivity [15]. For instance, Zn deficiency in the soil reduces yield by up to $13 \%$. $\mathrm{Zn}$ and $\mathrm{Mg}$ largely increase crop 
yields. $\mathrm{Zn}$ is involved in many physiological functions, which positively impact groundnut crop yields at low concentrations [16]. [17] showed that $\mathrm{Zn}$ application results in a remarkable increase of groundnut yield. It is an active element in the biochemical processes [18] and interferes chemically and biologically with $\mathrm{N}$ and $\mathrm{P}$ [19].

Likewise, $\mathrm{Mg}$ also promotes the uptake of $\mathrm{N}$ and $\mathrm{K}$ fertilizers [20]. Mg also promotes assimilation and migration of $\mathrm{P}$ in the plant and seed grains to form phytine and lipid [21]. According to these authors, reasonable application of $\mathrm{P}$ and $\mathrm{K}$ fertilizers with an appropriate supply of magnesium sulphate has significant effect on plant growth and nutrient uptake and groundnut yield improvement. Furthermore, [21] holds that $\mathrm{Mg}$ combined with $\mathrm{P}$ increase groundnut yield by $69.3 \%$ over the control plot. Similarly, [22] also showed that application of $\mathrm{Mg}$ and $\mathrm{P}$ increased groundnut yield by $12.7 \%$ compared to $\mathrm{P}$ application alone.

The aforementioned results show that balanced nutrition involving micronutrients and macronutrients is important to significantly induce groundnut yields while maintaining soil fertility. Despite the importance of these nutrients for balanced groundnut nutrition, no study has documented groundnut fertilization, with regards to $\mathrm{Zn}$ and $\mathrm{Mg}$ in Benin soils. Groundnut is a crop of high economic importance, but it is cultivated without application of mineral fertilizer leading to soil mining, because a large part of nutrients is in the seeds [23].

The aim of the present study was to assess the response of groundnut to different doses of N, P, K, Mg and $\mathrm{Zn}$ for optimal grain yield in the Sudano-Guinean and Guinean zones of Benin. Specifically, the effect of these nutrient doses on plant growth (root length, number of gynophores, number of nodules and plant recovery) and yield components (pod and aboveground biomass production) are assessed and nutrient optimal doses are determined. We assume that synergistic interaction among $\mathrm{N}, \mathrm{P}, \mathrm{K}, \mathrm{Mg}$ and $\mathrm{Zn}$ could optimize groundnut pod yield.

\section{Materials and Methods}

\subsection{Study Area}

The study was carried out in the municipalities of Bembèrèkè and Ouessè located in the southern Borgou agroecological zone (AEZ 3) and in the cotton agroecological zone (AEZ 5) of the centre of Benin respectively.

The AEZ 3 is located between $1^{\circ} 10^{\prime} \mathrm{E}-3^{\circ} 45^{\prime} \mathrm{E}$ and $9^{\circ} 45^{\prime} \mathrm{N}-12^{\circ} 25^{\prime} \mathrm{N}$. This zone is characterized by a unimodal rainfall distribution, with an average annual rainfall less than $1000 \mathrm{~mm}$ and located in the Sudanian zone. The relative moisture varies from $18 \%$ to $99 \%$ while temperature varies between $24^{\circ} \mathrm{C}$ and $31^{\circ} \mathrm{C}$. The Ferric and Plintic Luvisol [24] are the dominant soil types. Maize, sorghum, millet, yam, and groundnut are annual crops, cotton and soybean are the main cash crops in the area.

The AEZ 5 is located between $1^{\circ} 45^{\prime} \mathrm{E}-2^{\circ} 24^{\prime} \mathrm{E}$ and $6^{\circ} 25^{\prime} \mathrm{N}-7^{\circ} 30^{\prime} \mathrm{N}$. The area is under the sudano-guinean zone also called transitional zone. The annual mean 
temperature varies between $26^{\circ} \mathrm{C}$ and $29^{\circ} \mathrm{C}$ and the average annual rainfall varies between 1000 to $1400 \mathrm{~mm}$. The relative moisture varies from 69\% to $97 \%$. The Ferric and Plintic Luvisol are also the dominant soil types in the area. Black and hydromorphic soils are also found in the rivers' valleys. Maize, yam, cassava and groundnut represent the annual crops, while cotton and soybean are also the main cash crops.

\subsection{Experimental Design and Field Experiments}

Two years (2018 and 2019) on-farm experiments were carried out during the growing season. The experimental design was a full factorial design consisting of 46 treatments (representing combinations of $\mathrm{N}, \mathrm{P}, \mathrm{K}, \mathrm{Mg}$ and $\mathrm{Zn}$ doses) and a control plot replicated in five farmers' fields. The Box and Behnken design was used to determine the different treatments tested. Three doses of each nutrient (0-20-30 kg.ha- for $\mathrm{N}$; 0-30-50 kg.ha ${ }^{-1}$ for P; 0-20-40 kg.ha ${ }^{-1}$ for $\mathrm{K}, 0-15-30$ $\mathrm{kg} \cdot \mathrm{ha}^{-1}$ for $\mathrm{Mg}$ and $0-4-8 \mathrm{~kg} \cdot \mathrm{ha}^{-1}$ for $\mathrm{Zn}$ ) were tested. Each factor was set at its mean coded level 0 and a factorial plan of $2 k(k-1)+C_{0}$, (with $k$ the number of factors and $C_{0}$ the number of central points) of points is constructed with the other factors using the minimum code -1 and maximum code +1 level of each of these factors. The different combinations of the 5 nutrient levels in each treatment were generated for the response surface plan with MINITAB 18 software.

The experimental units were $5 \mathrm{~m} \times 4 \mathrm{~m}\left(20 \mathrm{~m}^{2}\right)$. Plots with previous maize crops were selected for the trial and managed by each farmer owner of the land. Groundnut variety sown was TS 32-1 called locally "Moto" (90 days of growth cycle with attainable yield in farmer's condition of $1.7 \mathrm{t} \cdot \mathrm{ha}^{-1}$ ). The seeds were purchased at CRA-Nord (Centre de Recherche-Agricole du Nord) of Benin National Agricultural Research Institute (INRAB) located at Ina in the north. The seed viability was around $80 \%$. Ridge ploughing was carried out with a $50 \mathrm{~cm}$ row spacing (in the Centre) and flat ploughing using animal traction with a depth of $15 \mathrm{~cm}$ (in the South Borgou zone). Sowing is carried out at a depth of 5 $\mathrm{cm}$ using two seeds per hole and $50 \mathrm{~cm}$ between rows and $20 \mathrm{~cm}$ between plants are the sowing spaces. Nutrients are applied in the form of urea $(46 \% \mathrm{~N})$, TSP $\left(46 \% \mathrm{P}_{2} \mathrm{O}_{5}\right), \mathrm{KCl}\left(60 \% \mathrm{~K}_{2} \mathrm{O}\right)$, kieserite $(23.5 \% \mathrm{MgO})$ and zinc sulphate $(35 \%$ $\left.\mathrm{Zn}^{2+}\right)$. Fertilizer application is carried out 15 days after sowing closed to each hole under the supervision of the research team and considering the doses calculated.

The collected data represent the recovery rate $(D)$, root length, number of gynophores and nodules, pod and aboveground biomass yields. $D$ (measured on 5 plants) is estimated on the basis of the following formula [25]:

$$
D=\pi / 4\left(D_{1}+D_{2} / 2\right)^{2}
$$

With $D_{1}$ and $D_{2}$ the perpendicular diameters measure when the sun is at the zenith. Root length (main root), number of gynophores and nodules were taken at full flowering, i.e. on the $60^{\text {th }}$ day after sowing on 10 selected plants from each experimental unit. Groundnut was harvested at maturity stage, when the leaves 
were dry. Seed samples and aboveground biomass were taken and sent to the laboratory for drying in an electric oven at $65^{\circ} \mathrm{C}$ for 72 hours to determine the dry matter.

Composite soil samples were taken before fertilizer application from nine sampling points in the experimental plots at $0-20 \mathrm{~cm}$ depth. Soil chemical analyses were carried out at the Laboratory of Soil Science, Water and Environment (LSSEE) of the National Agricultural Research Institute of Benin (LSSEE/INRAB). Analyses included particle size distribution (by sieve and Robison pipette method after removal of organic matter, carbonates and iron oxides), $\mathrm{pH}$ (water) using a glass electrode in 1:2.5 v/v soil solution, organic carbon according to Walkley and Black method, total nitrogen according to Kjeldahl digestion method in a mixture of $\mathrm{H}_{2} \mathrm{SO}_{4}$ and selenium followed by distillation and titration, phosphorus according to Bray 1 method, exchangeable $\mathrm{K}^{+}$and cations exchange capacity (CEC) in $1 \mathrm{~N}$ ammonium acetate at $\mathrm{pH} 7$ method after which $\mathrm{K}^{+}$was determined with a flame photometer.

\subsection{Statistical Analysis}

The statistical analyses were performed using SAS v. 9.4 packages. The collected data in each agroecological zone were subjected to one-way analysis of variance considering the treatments. General linear mixed-effect model, considering farmers as a random factor and nutrient combinations as a fixed factor. Student Newman-Keuls test was performed for means separation at a significance levels of $\mathrm{P}<0.05$.

For the optimal dose determination, the response surface analysis method was used [26]. The overall equation of the regression models for the response surface analysis was:

$$
y=\beta_{0}+\sum_{i=1}^{k} \beta_{i} x_{i}+\sum_{i=1}^{k} \beta_{i i} x_{i}^{2}+\sum_{1 \leq i \leq j}^{k} \beta_{i j} x_{i j}+\varepsilon
$$

where $y, x_{i}$ and $x_{\mathrm{j}}$ are the factors, $\beta_{0}$ is the constant or intercept, $\beta_{i}, \beta_{i i}$ and $\beta_{i j}$ represent the first-order, quadratic and interaction term coefficients, respectively, and $\varepsilon$ is the residual associated with the experiments.

The maximum fertilizer doses are obtained by cancelling out the first derivative of the above equation with respect to each fertilizer unit (the marginal productivity of the fertilizer) [26]. Thus, solving this system of equations helps to determine the values of $x_{1}, x_{2}, x_{3}, x_{4}$ and $x_{5}$ which were the maximum doses of each nutrient. $P_{Y}$ was the price of the product and $P_{\mathrm{N}}, P_{\mathrm{P}}, P_{\mathrm{K}}, P_{\mathrm{Mg}}$ and $P_{\mathrm{Zn}}$ the respective prices of kilogram of $\mathrm{N}, \mathrm{P}, \mathrm{K}, \mathrm{Mg}$ and $\mathrm{Zn}$. The maximum profit was obtained by equating the marginal product to the fertilizer/product price ratio as follow:

$$
\Delta y / \Delta x_{i}=P_{N} / P_{Y}
$$

The fixed doses that provide the maximum benefit represent the optimal doses of each nutrient. 


\section{Results}

\subsection{Soil Physico-Chemicals Parameters}

The soil particle sizes vary from sandy to sandy clayey texture. The $\mathrm{pH}$ (water) were 6.5 and 6.25 for the sites of Ouessè and Bembèrèkè respectively; soil organic $\mathrm{C}$ were 6.2 and $4.8 \mathrm{~g} \cdot \mathrm{kg}^{-1}$ for Ouessè and Bembèrèkè respectively; total $\mathrm{N}$ were 0.89 and $0.41 \mathrm{~g} \cdot \mathrm{kg}^{-1}$ for Ouessè and Bembèrèkè respectively; available $\mathrm{P}$ are 45.25 and $14.25 \mathrm{mg} \cdot \mathrm{kg}^{-1}$ for Ouèssè and Bembèrèkè respectively and the exchangeable $\mathrm{K}^{+}$were 0.34 and $0.18 \mathrm{cmol} \cdot \mathrm{kg}^{-1}$ for Ouessè and Bembèrèkè respectively. The cation exchange capacity (CEC) of both soils were low $\left(<15 \mathrm{cmol} \cdot \mathrm{kg}^{-1}\right)$. In general, the soils of the study area are slightly acid with low organic matter content (with $\mathrm{C} / \mathrm{N}$ ratios varying between 10 and 14). The consequence of this low $\mathrm{C} / \mathrm{N}$ ratio is a low level of the total $\mathrm{N}$ which seems to be with $\mathrm{P}$, the most limiting nutrients for both soils. Overall, the soils of the site are characterized by a low fertility level.

\subsection{Effect of the Different Nutrients N, P, K, Mg and Zn and Their Doses Applied on Groundnut Growth Parameters}

The growth parameters (plant recovery diameter, number of nodules and number of gynophores) induced by the treatments are shown in Table 1 and plant root lengths in Figure 1. The analysis of variance reveals that root length,

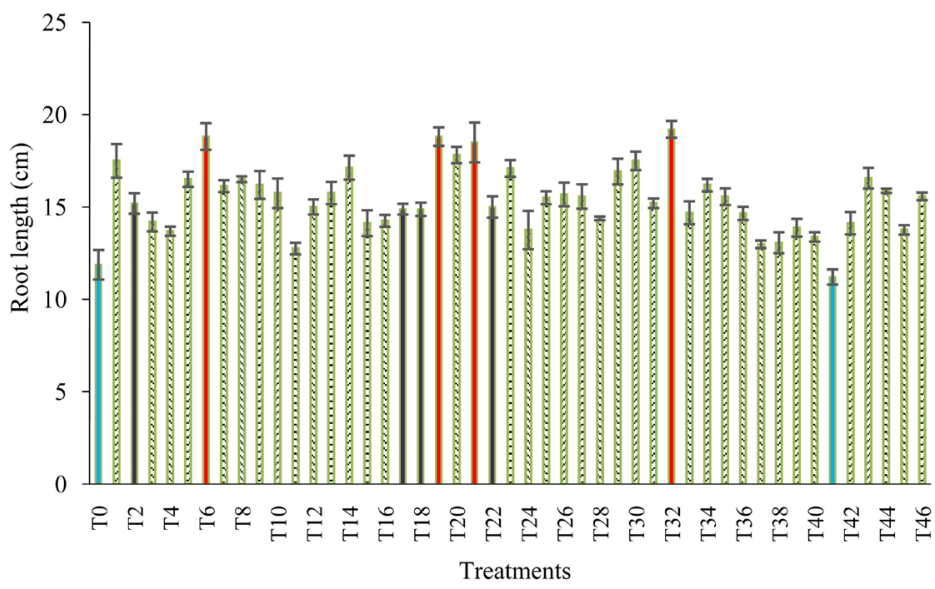

Figure 1. Average values of groundnut root lengths regarding the different treatments. $\mathrm{T}_{0}$ $=\mathrm{N}_{0} \mathrm{P}_{0} \mathrm{~K}_{0} \mathrm{Mg}_{0} \mathrm{Zn}_{0} ; \mathrm{T}_{1}=\mathrm{N}_{20} \mathrm{P}_{0} \mathrm{~K}_{20} \mathrm{Mg}_{0} \mathrm{Zn}_{4} ; \mathrm{T}_{2}=\mathrm{N}_{20} \mathrm{P}_{50} \mathrm{~K}_{20} \mathrm{Mg}_{15} \mathrm{Zn}_{8} ; \mathrm{T}_{3}=\mathrm{N}_{40} \mathrm{P}_{25} \mathrm{~K}_{20} \mathrm{Mg}_{15} \mathrm{Zn}_{0} ; \mathrm{T}_{4}$ $=\mathrm{N}_{20} \mathrm{P}_{25} \mathrm{~K}_{0} \mathrm{Mg}_{15} \mathrm{Zn}_{0} ; \mathrm{T}_{5}=\mathrm{N}_{20} \mathrm{P}_{25} \mathrm{~K}_{20} \mathrm{Mg}_{0} \mathrm{Zn}_{8} ; \mathrm{T}_{6}=\mathrm{N}_{40} \mathrm{P}_{25} \mathrm{~K}_{20} \mathrm{Mg}_{30} \mathrm{Zn}_{4} ; \mathrm{T}_{7}=\mathrm{N}_{0} \mathrm{P}_{25} \mathrm{~K}_{20} \mathrm{Mg}_{30} \mathrm{Zn}_{4} ; \mathrm{T}_{8}=$ $\mathrm{N}_{20} \mathrm{P}_{50} \mathrm{~K}_{40} \mathrm{Mg}_{15} \mathrm{Zn}_{4} ; \mathrm{T}_{9}=\mathrm{N}_{0} \mathrm{P}_{25} \mathrm{~K}_{20} \mathrm{Mg}_{0} \mathrm{Zn}_{4} ; \mathrm{T}_{10}=\mathrm{N}_{20} \mathrm{P}_{25} \mathrm{~K}_{40} \mathrm{Mg}_{0} \mathrm{Zn}_{4} ; \mathrm{T}_{11}=\mathrm{N}_{0} \mathrm{P}_{0} \mathrm{~K}_{20} \mathrm{Mg}_{15} \mathrm{Zn}_{4}$; $\mathrm{T}_{12}=\mathrm{N}_{20} \mathrm{P}_{25} \mathrm{~K}_{20} \mathrm{Mg}_{30} \mathrm{Zn}_{8} ; \mathrm{T}_{13}=\mathrm{N}_{0} \mathrm{P}_{50} \mathrm{~K}_{20} \mathrm{Mg}_{15} \mathrm{Zn}_{4} ; \mathrm{T}_{14}=\mathrm{N}_{20} \mathrm{P}_{0} \mathrm{~K}_{20} \mathrm{Mg}_{15} \mathrm{Zn}_{8} ; \mathrm{T}_{15}=\mathrm{N}_{20} \mathrm{P}_{25} \mathrm{~K}_{40} \mathrm{Mg}_{30} \mathrm{Zn}_{4} ;$ $\mathrm{T}_{16}=\mathrm{N}_{0} \mathrm{P}_{25} \mathrm{~K}_{0} \mathrm{Mg}_{15} \mathrm{Zn}_{4} ; \mathrm{T}_{17}=\mathrm{N}_{20} \mathrm{P}_{0} \mathrm{~K}_{0} \mathrm{Mg}_{15} \mathrm{Zn}_{4} ; \mathrm{T}_{18}=\mathrm{N}_{40} \mathrm{P}_{25} \mathrm{~K}_{20} \mathrm{Mg}_{15} \mathrm{Zn}_{8} ; \mathrm{T}_{19}=\mathrm{N}_{40} \mathrm{P}_{25} \mathrm{~K}_{40} \mathrm{Mg}_{15} \mathrm{Zn}_{4} ;$ $\mathrm{T}_{20}=\mathrm{N}_{20} \mathrm{P}_{50} \mathrm{~K}_{20} \mathrm{Mg}_{30} \mathrm{Zn}_{4} ; \mathrm{T}_{21}=\mathrm{N}_{20} \mathrm{P}_{0} \mathrm{~K}_{40} \mathrm{Mg}_{15} \mathrm{Zn}_{4} ; \mathrm{T}_{22}=\mathrm{N}_{20} \mathrm{P}_{25} \mathrm{~K}_{40} \mathrm{Mg}_{15} \mathrm{Zn}_{8} ; \mathrm{T}_{23}=\mathrm{N}_{20} \mathrm{P}_{25} \mathrm{~K}_{0} \mathrm{Mg}_{15} \mathrm{Zn}_{8} ;$ $\mathrm{T}_{24}=\mathrm{N}_{0} \mathrm{P}_{25} \mathrm{~K}_{40} \mathrm{Mg}_{15} \mathrm{Zn}_{4} ; \mathrm{T}_{25}=\mathrm{N}_{20} \mathrm{P}_{25} \mathrm{~K}_{0} \mathrm{Mg}_{0} \mathrm{Zn}_{4} ; \mathrm{T}_{26}=\mathrm{N}_{20} \mathrm{P}_{0} \mathrm{~K}_{20} \mathrm{Mg}_{30} \mathrm{Zn}_{4} ; \mathrm{T}_{27}=\mathrm{N}_{20} \mathrm{P}_{25} \mathrm{~K}_{20} \mathrm{Mg}_{15} \mathrm{Zn} 4 ;$ $\mathrm{T}_{28}=\mathrm{N}_{0} \mathrm{P}_{25} \mathrm{~K}_{20} \mathrm{Mg}_{15} \mathrm{Zn}_{0} ; \mathrm{T}_{29}=\mathrm{N}_{20} \mathrm{P}_{25} \mathrm{~K}_{20} \mathrm{Mg}_{30} \mathrm{Zn}_{0} ; \mathrm{T}_{30}=\mathrm{N}_{20} \mathrm{P}_{25} \mathrm{~K}_{40} \mathrm{Mg}_{15} \mathrm{Zn}_{0} ; \mathrm{T}_{31}=\mathrm{N}_{20} \mathrm{P}_{50} \mathrm{~K}_{20} \mathrm{Mg}_{0} \mathrm{Zn}_{4} ;$ $\mathrm{T}_{32}=\mathrm{N}_{40} \mathrm{P}_{0} \mathrm{~K}_{20} \mathrm{Mg}_{15} \mathrm{Zn}_{4} ; \mathrm{T}_{33}=\mathrm{N}_{20} \mathrm{P}_{25} \mathrm{~K}_{20} \mathrm{Mg}_{15} \mathrm{Zn}_{4} ; \mathrm{T}_{34}=\mathrm{N}_{20} \mathrm{P}_{25} \mathrm{~K}_{20} \mathrm{Mg}_{15} \mathrm{Zn}_{4} ; \mathrm{T}_{35}=\mathrm{N}_{20} \mathrm{P}_{25} \mathrm{~K}_{20} \mathrm{Mg}_{15} \mathrm{Zn}_{4} ;$ $\mathrm{T}_{36}=\mathrm{N}_{20} \mathrm{P}_{25} \mathrm{~K}_{20} \mathrm{Mg}_{0} \mathrm{Zn}_{0} ; \mathrm{T}_{37}=\mathrm{N}_{0} \mathrm{P}_{25} \mathrm{~K}_{20} \mathrm{Mg}_{15} \mathrm{Zn}_{8} ; \mathrm{T}_{38}=\mathrm{N}_{20} \mathrm{P}_{0} \mathrm{~K}_{20} \mathrm{Mg}_{15} \mathrm{Zn}_{0} ; \mathrm{T}_{39}=\mathrm{N}_{20} \mathrm{P}_{50} \mathrm{~K}_{0} \mathrm{Mg}_{15} \mathrm{Zn}_{4} ;$ $\mathrm{T}_{40}=\mathrm{N}_{40} \mathrm{P}_{25} \mathrm{~K}_{0} \mathrm{Mg}_{15} \mathrm{Zn}_{4} ; \mathrm{T}_{41}=\mathrm{N}_{40} \mathrm{P}_{50} \mathrm{~K}_{20} \mathrm{Mg}_{15} \mathrm{Zn}_{4} ; \mathrm{T}_{42}=\mathrm{N}_{20} \mathrm{P}_{25} \mathrm{~K}_{20} \mathrm{Mg}_{15} \mathrm{Zn}_{4} ; \mathrm{T}_{43}=\mathrm{N}_{20} \mathrm{P}_{25} \mathrm{~K}_{0} \mathrm{Mg}_{30} \mathrm{Zn}_{4} ;$ $\mathrm{T}_{44}=\mathrm{N}_{20} \mathrm{P}_{25} \mathrm{~K}_{20} \mathrm{Mg}_{15} \mathrm{Zn}_{4} ; \mathrm{T}_{45}=\mathrm{N}_{40} \mathrm{P}_{25} \mathrm{~K}_{20} \mathrm{Mg}_{0} \mathrm{Zn}_{4} ; \mathrm{T}_{46}=\mathrm{N}_{20} \mathrm{P}_{50} \mathrm{~K}_{20} \mathrm{Mg}_{15} \mathrm{Zn}_{0}$. 
Table 1. Mean values ( \pm standard errors) of plant recovery diameter, number of nodules and number of gynophores regarding the treatments and periods of measurement.

\begin{tabular}{|c|c|c|c|c|c|}
\hline \multirow{2}{*}{ Treatments } & \multicolumn{3}{|c|}{ Recovery diameter $\left(\mathrm{cm}^{2}\right)$} & \multirow{2}{*}{$\begin{array}{l}\text { Number of } \\
\text { gynophores }\end{array}$} & \multirow{2}{*}{$\begin{array}{l}\text { Number of } \\
\text { nodules }\end{array}$} \\
\hline & $30 \mathrm{DAS}$ & $50 \mathrm{DAS}$ & 70 DAS & & \\
\hline $\mathrm{N}_{0} \mathrm{P}_{0} \mathrm{~K}_{0} \mathrm{Mg}_{0} \mathrm{Zn}_{0}$ & $179.5 \pm 9.2 \mathrm{u}$ & $759.2 \pm 18 \mathrm{~s}$ & $1262 \pm 23 r$ & $15.1 \pm 1.1 \mathrm{r}$ & $54.7 \pm 9.7 \mathrm{t}$ \\
\hline $\mathrm{N}_{20} \mathrm{P}_{0} \mathrm{~K}_{20} \mathrm{Mg}_{0} \mathrm{Zn}_{4}$ & $378.4 \pm 9.4 \mathrm{jkl}$ & $1126.6 \pm 6.9 \mathrm{ijk}$ & $1724.1 \pm 21.1 \mathrm{hij}$ & $27.8 \pm 2.2 \mathrm{opq}$ & $158.7 \pm 3.8 \mathrm{opq}$ \\
\hline $\mathrm{N}_{20} \mathrm{P}_{50} \mathrm{~K}_{20} \mathrm{Mg}_{15} \mathrm{Zn}_{8}$ & $367.9 \pm 29.3 \mathrm{mno}$ & $1111 \pm 51 \mathrm{klm}$ & $1705.7 \pm 63.2 \mathrm{jkl}$ & $34.1 \pm 2.8 \mathrm{kl}$ & $159.5 \pm 17.3 \mathrm{opq}$ \\
\hline $\mathrm{N}_{40} \mathrm{P}_{25} \mathrm{~K}_{20} \mathrm{Mg}_{15} \mathrm{Zn}_{0}$ & $388.2 \pm 11.3 \mathrm{ghi}$ & $1147.3 \pm 20.4$ fgh & $1750.9 \pm 25.5 \mathrm{fgh}$ & $33.9 \pm 2.1 \mathrm{klm}$ & $202.7 \pm 9.8 \mathrm{jk}$ \\
\hline $\mathrm{N}_{20} \mathrm{P}_{25} \mathrm{~K}_{0} \mathrm{Mg}_{15} \mathrm{Zn}_{0}$ & $395.7 \pm 15.6 \mathrm{def}$ & $1156.4 \pm 26.8 \mathrm{def}$ & $1761 \pm 33.2 \mathrm{def}$ & $42.25 \pm 1.8 \mathrm{bcd}$ & $173 \pm 10.3 \mathrm{mn}$ \\
\hline $\mathrm{N}_{20} \mathrm{P}_{25} \mathrm{~K}_{20} \mathrm{Mg}_{0} \mathrm{Zn}_{8}$ & $337.9 \pm 11.7 \mathrm{rst}$ & $1057.5 \pm 21.9 \mathrm{pqr}$ & $1639 \pm 27.6 o p q$ & $25.2 \pm 0.8 \mathrm{q}$ & $158.5 \pm 20$ nopq \\
\hline $\mathrm{N}_{40} \mathrm{P}_{25} \mathrm{~K}_{20} \mathrm{Mg}_{30} \mathrm{Zn}_{4}$ & $373.9 \pm 7.81 \mathrm{mn}$ & $1120.4 \pm 14.4 \mathrm{jkl}$ & $1717 \pm 18.2 \mathrm{ijk}$ & $24.8 \pm 2.1 \mathrm{q}$ & $173.8 \pm 18.3 \mathrm{mn}$ \\
\hline $\mathrm{N}_{0} \mathrm{P}_{25} \mathrm{~K}_{20} \mathrm{Mg}_{30} \mathrm{Zn}_{4}$ & $316 \pm 13.8 \mathrm{rst}$ & $1018.9 \pm 25.6 \mathrm{pqr}$ & $1591 \pm 32.2 \mathrm{opq}$ & $31.6 \pm 2.8 \operatorname{lmn}$ & $236 \pm 27 \mathrm{~h}$ \\
\hline $\mathrm{N}_{20} \mathrm{P}_{50} \mathrm{~K}_{40} \mathrm{Mg}_{15} \mathrm{Zn}_{4}$ & $464.1 \pm 10.6 \mathrm{a}$ & $1275.1 \pm 17.8 \mathrm{a}$ & $1908 \pm 21.9 \mathrm{a}$ & $26.5 \pm 1.2 \mathrm{pq}$ & $167.5 \pm 9.3 \mathrm{mno}$ \\
\hline $\mathrm{N}_{0} \mathrm{P}_{25} \mathrm{~K}_{20} \mathrm{Mg}_{0} \mathrm{Zn}_{4}$ & $309.6 \pm 14.8 \mathrm{rst}$ & $1006.7 \pm 29.1 \mathrm{pqr}$ & $1575.5 \pm 37.2 \mathrm{opq}$ & $25.6 \pm 3.3 q$ & $216.5 \pm 16.2 \mathrm{ij}$ \\
\hline $\mathrm{N}_{20} \mathrm{P}_{25} \mathrm{~K}_{40} \mathrm{Mg}_{0} \mathrm{Zn}_{4}$ & $406.1 \pm 16.2 \mathrm{bde}$ & $1176 \pm 24.3 \mathrm{bcd}$ & $1785.8 \pm 28.8 \mathrm{bcd}$ & $35.6 \pm 2.8 \mathrm{ijk}$ & $312.8 \pm 6.2 \mathrm{~d}$ \\
\hline $\mathrm{N}_{0} \mathrm{P}_{0} \mathrm{~K}_{20} \mathrm{Mg}_{15} \mathrm{Zn}_{4}$ & $361.5 \pm 25.2 \mathrm{rst}$ & $1097.9 \pm 41.7 \mathrm{mn}$ & $1688.9 \pm 50.9 \mathrm{klm}$ & $40.3 \pm 1.8 \mathrm{cdef}$ & $295 \pm 20.5 \mathrm{e}$ \\
\hline $\mathrm{N}_{20} \mathrm{P}_{25} \mathrm{~K}_{20} \mathrm{Mg}_{30} \mathrm{Zn}_{8}$ & $363.5 \pm 13.9$ nop & $1101.6 \pm 21.4 \mathrm{lmn}$ & $1693.5 \pm 25.7 \mathrm{klm}$ & $35.5 \pm 2.5 \mathrm{ijk}$ & $163.1 \pm 2.2 \mathrm{nopq}$ \\
\hline $\mathrm{N}_{0} \mathrm{P}_{50} \mathrm{~K}_{20} \mathrm{Mg}_{15} \mathrm{Zn}_{4}$ & $417.5 \pm 13.1 \mathrm{~b}$ & $1195.8 \pm 20.9 b$ & $1810.3 \pm 25.4 \mathrm{~b}$ & $29.2 \pm 1$ nop & $162.5 \pm 7.5 \mathrm{nopq}$ \\
\hline $\mathrm{N}_{20} \mathrm{P}_{0} \mathrm{~K}_{20} \mathrm{Mg}_{15} \mathrm{Zn}_{8}$ & $390 \pm 27.4 \mathrm{cbd}$ & $1149.8 \pm 47.6 \mathrm{efg}$ & $1753.9 \pm 58.9 \mathrm{efg}$ & $39.2 \pm 2.9 \mathrm{defg}$ & $334.8 \pm 16.6 \mathrm{c}$ \\
\hline $\mathrm{N}_{20} \mathrm{P}_{25} \mathrm{~K}_{40} \mathrm{Mg}_{30} \mathrm{Zn}_{4}$ & $400 \pm 25 \mathrm{def}$ & $1167.4 \pm 43.2 \mathrm{cde}$ & $1775.7 \pm 53.4 \mathrm{cde}$ & $43.88 \pm 3.25 b$ & $390 \pm 12.8 \mathrm{a}$ \\
\hline $\mathrm{N}_{0} \mathrm{P}_{25} \mathrm{~K}_{0} \mathrm{Mg}_{15} \mathrm{Zn}_{4}$ & $335.9 \pm 13.8 \mathrm{rst}$ & $1055.6 \pm 25.2 \mathrm{pqr}$ & $1637.1 \pm 31.60 \mathrm{pq}$ & $37.3 \pm 1.1 \mathrm{fghij}$ & $162 \pm 19.4$ nopq \\
\hline $\mathrm{N}_{20} \mathrm{P}_{0} \mathrm{~K}_{0} \mathrm{Mg}_{15} \mathrm{Zn}_{4}$ & $390.4 \pm 9.2 \mathrm{efg}$ & $1143.6 \pm 19.2 \mathrm{fgh}$ & $1743.9 \pm 24.9 \mathrm{ghi}$ & $44 \pm 3.3 b$ & $232.8 \pm 18.1 \mathrm{hi}$ \\
\hline $\mathrm{N}_{40} \mathrm{P}_{25} \mathrm{~K}_{20} \mathrm{Mg}_{15} \mathrm{Zn}_{8}$ & $330.9 \pm 141 \mathrm{mn}$ & $1045.5 \pm 22.1 \mathrm{pqr}$ & $1624.2 \pm 26.6 \mathrm{opq}$ & $39.6 \pm 2.1 \mathrm{cdef}$ & $163 \pm 6.5$ nopq \\
\hline $\mathrm{N}_{40} \mathrm{P}_{25} \mathrm{~K}_{40} \mathrm{Mg}_{15} \mathrm{Zn}_{4}$ & $346 \pm 17 \mathrm{rst}$ & $1072.3 \pm 30.3 \mathrm{opq}$ & $1657.6 \pm 37.8 \mathrm{nop}$ & $30.9 \pm 1.6 \mathrm{mno}$ & $263.6 \pm 15.2 f$ \\
\hline $\mathrm{N}_{20} \mathrm{P}_{50} \mathrm{~K}_{20} \mathrm{Mg}_{30} \mathrm{Zn}_{4}$ & $370.4 \pm 14.8 \mathrm{mno}$ & $1116.1 \pm 25.2 \mathrm{klm}$ & $1712.2 \pm 31.1 \mathrm{jkl}$ & $41.4 \pm 2.2 \mathrm{bcde}$ & $244.6 \pm 12.8 \mathrm{gh}$ \\
\hline $\mathrm{N}_{20} \mathrm{P}_{0} \mathrm{~K}_{40} \mathrm{Mg}_{15} \mathrm{Zn}_{4}$ & $323.3 \pm 18.5 \mathrm{rst}$ & $1032 \pm 31.5 \mathrm{pqr}$ & $1607.4 \pm 38.8 \mathrm{lopq}$ & $40.6 \pm 1.5 \mathrm{cde}$ & $148.6 \pm 10.9 p q$ \\
\hline $\mathrm{N}_{20} \mathrm{P}_{25} \mathrm{~K}_{40} \mathrm{Mg}_{15} \mathrm{Zn}_{8}$ & $327.9 \pm 12.1 \mathrm{rst}$ & $1041.7 \pm 21.2 \mathrm{pqr}$ & $1619.9 \pm 26.3 \mathrm{opq}$ & $38.7 \pm 2.2 \mathrm{efgh}$ & $167.6 \pm 7 \mathrm{mno}$ \\
\hline $\mathrm{N}_{20} \mathrm{P}_{25} \mathrm{~K}_{0} \mathrm{Mg}_{15} \mathrm{Zn}_{8}$ & $325 \pm 21.4 \mathrm{rst}$ & $1033.7 \pm 38.8 \mathrm{pqr}$ & $1609.1 \pm 48.7 \mathrm{opq}$ & $41.4 \pm 2.9 \mathrm{bcde}$ & $164 \pm 8.7$ nop \\
\hline $\mathrm{N}_{0} \mathrm{P}_{25} \mathrm{~K}_{40} \mathrm{Mg}_{15} \mathrm{Zn}_{4}$ & $358.7 \pm 14.2 \mathrm{rst}$ & $1095.1 \pm 25.1 \mathrm{mno}$ & $1686.1 \pm 31.31 \mathrm{lmn}$ & $38.3 \pm 3.3$ efghi & $199.1 \pm 14.4 \mathrm{kl}$ \\
\hline $\mathrm{N}_{20} \mathrm{P}_{25} \mathrm{~K}_{0} \mathrm{Mg}_{0} \mathrm{Zn}_{4}$ & $358.2 \pm 11 \mathrm{rst}$ & $1094.6 \pm 19.7 \mathrm{mno}$ & $1685.5 \pm 24.6 \mathrm{lmn}$ & $42.3 \pm 3.4 \mathrm{bc}$ & $183.5 \pm 31 \mathrm{~m}$ \\
\hline $\mathrm{N}_{20} \mathrm{P}_{0} \mathrm{~K}_{20} \mathrm{Mg}_{30} \mathrm{Zn}_{4}$ & $296.1 \pm 17.9 t$ & $982.3 \pm 34.4 \mathrm{r}$ & $1544.9 \pm 43.7 \mathrm{q}$ & $24.8 \pm 5 q$ & $202.6 \pm 18.4 \mathrm{jk}$ \\
\hline $\mathrm{N}_{20} \mathrm{P}_{25} \mathrm{~K}_{20} \mathrm{Mg}_{15} \mathrm{Zn}_{4}$ & $354.3 \pm 15.6 \mathrm{rst}$ & $1088.2 \pm 27.1 \mathrm{nop}$ & $1677.7 \pm 33.5 \mathrm{mno}$ & $39.13 \pm 5.4 \mathrm{efg}$ & $124.3 \pm 13.9 \mathrm{r}$ \\
\hline $\mathrm{N}_{0} \mathrm{P}_{25} \mathrm{~K}_{20} \mathrm{Mg}_{15} \mathrm{Zn}_{0}$ & $386.8 \pm 16.9 \mathrm{ghi}$ & $1145 \pm 29.3 \mathrm{fgh}$ & $1748.2 \pm 36.2 \mathrm{fgh}$ & $33.6 \pm 2.4 \mathrm{klm}$ & $88.1 \pm 8.1 \mathrm{~s}$ \\
\hline $\mathrm{N}_{20} \mathrm{P}_{25} \mathrm{~K}_{20} \mathrm{Mg}_{30} \mathrm{Zn}_{0}$ & $385.7 \pm 13.6 \mathrm{hij}$ & $1142.2 \pm 22.6 \mathrm{ghi}$ & $1744.4 \pm 27.7 \mathrm{ghi}$ & $48.1 \pm 3 a$ & $313.1 \pm 16.9 \mathrm{~d}$ \\
\hline $\mathrm{N}_{20} \mathrm{P}_{25} \mathrm{~K}_{40} \mathrm{Mg}_{15} \mathrm{Zn}_{0}$ & $340 \pm 12.6 \mathrm{rst}$ & $1062.1 \pm 21.5 \mathrm{pqr}$ & $1645.1 \pm 26.4 \mathrm{opq}$ & $26.2 \pm 1.2 \mathrm{pq}$ & $360.6 \pm 7.8 b$ \\
\hline $\mathrm{N}_{20} \mathrm{P}_{50} \mathrm{~K}_{20} \mathrm{Mg}_{0} \mathrm{Zn}_{4}$ & $303.9 \pm 16.8 \mathrm{st}$ & $997.6 \pm 30.8 \mathrm{qr}$ & $1564.5 \pm 38.7 \mathrm{pq}$ & $31.6 \pm 3.31 \mathrm{lmn}$ & $207.6 \pm 15.5 \mathrm{jk}$ \\
\hline $\mathrm{N}_{40} \mathrm{P}_{0} \mathrm{~K}_{20} \mathrm{Mg}_{15} \mathrm{Zn}_{4}$ & $329.8 \pm 13.6 \mathrm{rst}$ & $1041.9 \pm 26.4 \mathrm{pqr}$ & $1619.2 \pm 33.6 \mathrm{opq}$ & $27.7 \pm 1 \mathrm{pq}$ & $192 \pm 11.5 \mathrm{kl}$ \\
\hline $\mathrm{N}_{20} \mathrm{P}_{25} \mathrm{~K}_{20} \mathrm{Mg}_{15} \mathrm{Zn}_{4}$ & $353.6 \pm 15.2 \mathrm{rst}$ & $1086.6 \pm 27 \mathrm{nop}$ & $1675.7 \pm 34.6 \mathrm{nop}$ & $25.7 \pm 1.8 \mathrm{q}$ & $248 \pm 11 \mathrm{fgh}$ \\
\hline $\mathrm{N}_{20} \mathrm{P}_{25} \mathrm{~K}_{20} \mathrm{Mg}_{15} \mathrm{Zn}_{4}$ & $311.8 \pm 17.5 \mathrm{rst}$ & $1011.3 \pm 31.3 \mathrm{pqr}$ & $1581.5 \pm 39.1 \mathrm{opq}$ & $33.5 \pm 1.7 \mathrm{klm}$ & $290.7 \pm 13.5 \mathrm{e}$ \\
\hline
\end{tabular}




\section{Continued}

\begin{tabular}{lccccc}
$\mathrm{N}_{20} \mathrm{P}_{25} \mathrm{~K}_{20} \mathrm{Mg}_{15} \mathrm{Zn}_{4}$ & $413.1 \pm 21 \mathrm{cb}$ & $1188 \pm 34.8 \mathrm{~b}$ & $1800.6 \pm 42.6 \mathrm{~b}$ & $36.3 \pm 2.9 \mathrm{ghijk}$ & $365.5 \pm 14.7 \mathrm{~b}$ \\
$\mathrm{~N}_{20} \mathrm{P}_{25} \mathrm{~K}_{20} \mathrm{Mg}_{0} \mathrm{Zn}_{0}$ & $402.8 \pm 15.4 \mathrm{cbd}$ & $1171.9 \pm 25.4 \mathrm{cde}$ & $1781.2 \pm 31 \mathrm{cde}$ & $32.3 \pm 1 \mathrm{~lm}$ & $123.1 \pm 8.9 \mathrm{r}$ \\
$\mathrm{N}_{0} \mathrm{P}_{25} \mathrm{~K}_{20} \mathrm{Mg}_{15} \mathrm{Zn}_{8}$ & $384.5 \pm 12.1 \mathrm{ijk}$ & $1139.4 \pm 22.2 \mathrm{hij}$ & $1740.7 \pm 27.9 \mathrm{ghi}$ & $26.4 \pm 1.2 \mathrm{pq}$ & $206.1 \pm 16.7 \mathrm{jk}$ \\
$\mathrm{N}_{20} \mathrm{P}_{0} \mathrm{~K}_{20} \mathrm{Mg}_{15} \mathrm{Zn}_{0}$ & $375 \pm 18.7 \mathrm{klm}$ & $1123.1 \pm 33.2 \mathrm{jkl}$ & $1720.6 \pm 41.4 \mathrm{ijk}$ & $41.1 \pm 1.5 \mathrm{bcde}$ & $232.3 \pm 8.8 \mathrm{hi}$ \\
$\mathrm{N}_{20} \mathrm{P}_{50} \mathrm{~K}_{0} \mathrm{Mg}_{15} \mathrm{Zn}_{4}$ & $341.1 \pm 11.1 \mathrm{rst}$ & $1064.9 \pm 20.2 \mathrm{opq}$ & $1648.8 \pm 25.3 \mathrm{nop}$ & $36 \pm 1.8 \mathrm{hijk}$ & $303 \pm 15 \mathrm{de}$ \\
$\mathrm{N}_{40} \mathrm{P}_{25} \mathrm{~K}_{0} \mathrm{Mg}_{15} \mathrm{Zn}_{4}$ & $383 \pm 13.8 \mathrm{ijk}$ & $1137 \pm 22.4 \mathrm{hij}$ & $1737.9 \pm 27.3 \mathrm{ghi}$ & $39.7 \pm 1.7 \mathrm{cdef}$ & $255.1 \pm 14.2 \mathrm{fg}$ \\
$\mathrm{N}_{40} \mathrm{P}_{50} \mathrm{~K}_{20} \mathrm{Mg}_{15} \mathrm{Zn}_{4}$ & $327 \pm 26.2 \mathrm{rst}$ & $1039 \pm 47.1 \mathrm{pqr}$ & $1616.2 \pm 58.8 \mathrm{opq}$ & $39 \pm 3.5 \mathrm{efgh}$ & $257 \pm 14.2 \mathrm{fg}$ \\
$\mathrm{N}_{20} \mathrm{P}_{25} \mathrm{~K}_{20} \mathrm{Mg}_{15} \mathrm{Zn}_{4}$ & $354.1 \pm 14.7 \mathrm{rst}$ & $1088 \pm 26.2 \mathrm{nop}$ & $1677.5 \pm 32.7 \mathrm{mno}$ & $42.5 \pm 3.1 \mathrm{bc}$ & $318 \pm 20.7 \mathrm{~cd}$ \\
$\mathrm{~N}_{20} \mathrm{P}_{25} \mathrm{~K}_{0} \mathrm{Mg}_{30} \mathrm{Zn}_{4}$ & $336 \pm 15.4 \mathrm{rst}$ & $1056.5 \pm 27.3 \mathrm{pqr}$ & $1638.5 \pm 33.9 \mathrm{opq}$ & $33.7 \pm 1.7 \mathrm{klm}$ & $146.1 \pm 27.5 \mathrm{q}$ \\
$\mathrm{N}_{20} \mathrm{P}_{25} \mathrm{~K}_{20} \mathrm{Mg}_{15} \mathrm{Zn}_{4}$ & $362.6 \pm 9.6 \mathrm{rst}$ & $1101.5 \pm 18.2 \mathrm{lmn}$ & $1693.8 \pm 23 \mathrm{klm}$ & $41.3 \pm 3.5 \mathrm{bcde}$ & $155.5 \pm 13.5 \mathrm{opq}$ \\
$\mathrm{N}_{40} \mathrm{P}_{25} \mathrm{~K}_{20} \mathrm{Mg}_{0} \mathrm{Zn}_{4}$ & $390.8 \pm 11.9 \mathrm{efg}$ & $1151.5 \pm 20.7 \mathrm{efg}$ & $1756.1 \pm 25.7 \mathrm{efg}$ & $38.9 \pm 2.9 \mathrm{efgh}$ & $164.7 \pm 19 \mathrm{nop}$ \\
$\mathrm{N}_{20} \mathrm{P}_{50} \mathrm{~K}_{20} \mathrm{Mg}_{15} \mathrm{Zn}_{0}$ & $410.2 \pm 19.9 \mathrm{cbd}$ & $1183.4 \pm 33.8 \mathrm{bc}$ & $1795.1 \pm 41.6 \mathrm{bc}$ & $34.4 \pm 3.4 \mathrm{jkl}$ & $174.2 \pm 10.3 \mathrm{mn}$ \\
\hline
\end{tabular}

In a column, means followed by the same alphabetic letters are not significantly different $(\mathrm{p}>0.05)$ according to Student Newman-Keuls test. DAS $=$ Days After Sowing.

number of nodules and number of gynophores vary significantly $(\mathrm{p}=0.000)$ depending on the treatments. Similarly, the plant recovery diameter was largely $(\mathrm{p}$ $=0.001$ ) influenced by the different treatments applied. From Figure 1, it appears that plant root development was sensitive to high $\mathrm{N}$ doses as well as to the combination of intermediate doses of $\mathrm{P}$. The shortest root lengths $(11.87 \mathrm{~cm}$ and $11.21 \mathrm{~cm})$ were recorded with treatments $\mathrm{T}_{0}$ and $\mathrm{T}_{41}\left(\mathrm{~N}_{40} \mathrm{P}_{50} \mathrm{~K}_{20} \mathrm{Mg}_{15} \mathrm{Zn}_{4}\right)$ respectively. The highest root lengths $(19.2 \mathrm{~cm}$ and $18.8 \mathrm{~cm})$ were recorded with treatments $\mathrm{T}_{32}\left(\mathrm{~N}_{40} \mathrm{P}_{0} \mathrm{~K}_{20} \mathrm{Mg}_{15} \mathrm{Zn}_{4}\right), \mathrm{T}_{6}\left(\mathrm{~N}_{20} \mathrm{P}_{25} \mathrm{~K}_{20} \mathrm{Mg}_{0} \mathrm{Zn}_{8}\right)$ and $\mathrm{T}_{20}\left(\mathrm{~N}_{20} \mathrm{P}_{50} \mathrm{~K}_{20} \mathrm{Mg}_{30} \mathrm{Zn}_{4}\right)$ respectively. In general, the control plot showed the lowest root length.

Regarding plant recovery diameters (Table 1), a gradual trends were observed. The lowest recovery diameters were recorded 30 days after sowing (DAS). Plant recovery diameters are 2.5 times higher 50 and 70 DAS compared to the first measurement. The lowest plant recovery diameter was recorded with the control treatment. Treatments with intermediate nutrient rates $\left(\mathrm{N}_{20} \mathrm{P}_{25} \mathrm{~K}_{20} \mathrm{Mg}_{15} \mathrm{Zn}_{4}\right.$ and $\mathrm{N}_{20} \mathrm{P}_{25} \mathrm{~K}_{20} \mathrm{Mg}_{0} \mathrm{Zn}_{8}$ ) induced plant recovery diameters 1.5 and 1.4 times higher than the control plot 50 and 70 DAS. Equally, treatments with high doses of nutrients induced the highest plant recovery diameter 70 DAS. In general, it was observed that nutrients applied have induced good groundnut plant growth.

It was observed also from the table that the highest number of nodules was recorded with treatment $\mathrm{N}_{20} \mathrm{P}_{25} \mathrm{~K}_{40} \mathrm{Mg}_{30} \mathrm{Zn}_{4}$ followed by treatments $\mathrm{N}_{20} \mathrm{P}_{25} \mathrm{~K}_{20} \mathrm{Mg}_{15} \mathrm{Zn}_{4}$ and $\mathrm{N}_{20} \mathrm{P}_{25} \mathrm{~K}_{40} \mathrm{Mg}_{15} \mathrm{Zn}_{0}$ with numbers of nodules 6.5 and 7.2 times higher than the control plot. The lowest number of nodules was recorded in the control plot. In a nutshell, the treatments with no $\mathrm{Zn}$ showed a low number of nodules compared to the remain treatments with $\mathrm{Zn}$.

The highest number of gynophores was obtained with treatment $\mathrm{N}_{20} \mathrm{P}_{25} \mathrm{~K}_{20} \mathrm{Mg}_{30} \mathrm{Zn}_{0}$ followed by treatments $\mathrm{N}_{20} \mathrm{P}_{0} \mathrm{~K}_{0} \mathrm{Mg}_{15} \mathrm{Zn}_{4}, \mathrm{~N}_{20} \mathrm{P}_{25} \mathrm{~K}_{40} \mathrm{Mg}_{30} \mathrm{Zn}_{4}, \mathrm{~N}_{20} \mathrm{P}_{25} \mathrm{~K}_{20} \mathrm{Mg}_{15} \mathrm{Zn}_{4}$, $\mathrm{N}_{20} \mathrm{P}_{25} \mathrm{~K}_{0} \mathrm{Mg}_{0} \mathrm{Zn}_{4}$ and $\mathrm{N}_{20} \mathrm{P}_{25} \mathrm{~K}_{0} \mathrm{Mg}_{15} \mathrm{Zn}_{0}$ with number of gynophores 2.8 and 2.9 times higher than the control plot. 


\subsection{Effect of the Different Nutrients N, P, K, Mg and $\mathrm{Zn}$ and Their Doses Applied on Groundnut Pod Yield and Aboveground Biomass}

The average pod yield values and the aboveground biomass in both sites are shown in Table 2 and Table 3 respectively. In general, in both sites, the lowest pod yields were recorded on the control plot. In 2018 and 2019, treatments containing intermediate doses of nutrients $\left(\mathrm{N}_{20} \mathrm{P}_{25} \mathrm{~K}_{20} \mathrm{Mg}_{15} \mathrm{Zn}_{4}\right)$ showed pod yields up to 2.6 - 2.9 times higher than that of the control plot in the Centre and up to 3.2 times in the site of the north. The same trends were observed with the aboveground biomass. On the north site, the pod yields were higher in 2019 than those recorded in 2018. Likewise, the treatments containing maximum $\mathrm{P}$ rate (50 $\mathrm{kg} \cdot \mathrm{ha}^{-1}$ ) induced low pod yields but high aboveground biomass. The same trends were observed with the treatments containing $\mathrm{N}$ dose of $40 \mathrm{~kg} \cdot \mathrm{ha}^{-1}$.

\subsection{Response of the Groundnut Plant to Each Nutrient Type and Doses Applied}

The response surface diagrams of each nutrient applied and the interaction between each nutrient are displayed in Figure 2. Considering $\mathrm{P}$ and $\mathrm{Zn}$, high pod yields (above $2 \mathrm{t} \cdot \mathrm{ha}^{-1}$ ) were recorded with $\mathrm{P}$ doses between 20 and $25 \mathrm{~kg} \cdot \mathrm{ha}^{-1}$ combined with $\mathrm{Zn}$ doses between 2 and $4 \mathrm{~kg} \cdot \mathrm{ha}^{-1}$. The interaction showed that the pod yield increases only with low and approximately intermediate doses of $\mathrm{Zn}$ and P. This yield decreases with a high dose supply of both nutrients. Thus, the supply of these nutrients at high doses induced depressive effect on groundnut pod yields. With regard to $\mathrm{Mg}$, high pod yields (above $2 \mathrm{t} \cdot \mathrm{ha}^{-1}$ ) were recorded with $\mathrm{P}$ doses varying between 20 and $30 \mathrm{~kg} \cdot \mathrm{ha}^{-1}$ combined with $\mathrm{Mg}$ doses varying between 5 and $20 \mathrm{~kg} \cdot \mathrm{ha}^{-1}$. An increase of groundnut pod yields was recorded only with $\mathrm{P}$ doses varying between 2 and $20 \mathrm{~kg} \cdot \mathrm{ha}^{-1}$ regarding $\mathrm{Mg}$ doses applied. Thus, the uptake of these two nutrients at high dose induced depressive effect on groundnut pod yields. Furthermore, a depressive effect on the groundnut pod yields was noticed with $\mathrm{K}$ doses higher than $25 \mathrm{~kg} \cdot \mathrm{ha}^{-1}$ combined with rate of $\mathrm{Mg}$ applied. The same observation was made with $\mathrm{N}$ with pronounced depressive effect on pod yields when the rate exceeds $15 \mathrm{~kg} \cdot \mathrm{ha}^{-1}$ even if intermediate rates of $\mathrm{Zn}$ and $\mathrm{Mg}$ were applied. The high doses of these two nutrients induced a decrease of groundnut pod yields regarding $\mathrm{Zn}$ and $\mathrm{Mg}$ rates. The interaction between $\mathrm{Mg}$ and $\mathrm{Zn}$ did not induce pod yields above $2 \mathrm{t} \cdot \mathrm{ha}^{-1}$.

Figure 3 and Figure 4 show the response curves of groundnut pod yields to each nutrient applied at different rates in both experimental sites. A normal response was observed for all nutrients, except for $\mathrm{K}$, which revealed no response. It was also noticed that, the responses were strong for $\mathrm{N}$ and $\mathrm{P}$ but weak for $\mathrm{Mg}$ and Zn. Similarly, Figure 4 shows strong responses for $\mathrm{N}$ and $\mathrm{P}$ but weak responses for $\mathrm{K}, \mathrm{Mg}$ and $\mathrm{Zn}$. On both sites, maximum pod yields vary between 2 and $2.5 \mathrm{t} \cdot \mathrm{ha}^{-1}$ and this maximum yield decreases beyond the intermediate nutrient doses. 
Table 2. Mean values ( \pm standard errors) of the aboveground biomass and pod yields of groundnut regarding the combined doses of N, P, K, Mg and Zn during 2018 and 2019 growing seasons on the Central-Benin site.

\begin{tabular}{|c|c|c|c|c|}
\hline \multirow{2}{*}{ Treatments } & \multicolumn{2}{|c|}{ Pod yields $\left(\mathrm{t} \cdot \mathrm{DM} \cdot \mathrm{ha}^{-1}\right)$} & \multicolumn{2}{|c|}{ Aboveground biomass $\left(\mathrm{t} \cdot \mathrm{DM} \cdot \mathrm{ha}^{-1}\right)$} \\
\hline & 2018 & 2019 & 2018 & 2019 \\
\hline $\mathrm{N}_{0} \mathrm{P}_{0} \mathrm{~K}_{0} \mathrm{Mg}_{0} \mathrm{Zn}_{0}$ & $0.68 \pm 0.01 \mathrm{u}$ & $0.71 \pm 0.07 \mathrm{w}$ & $0.7 \pm 0.02 \mathrm{r}$ & $0.66 \pm 0.05 \mathrm{~s}$ \\
\hline $\mathrm{N}_{20} \mathrm{P}_{0} \mathrm{~K}_{20} \mathrm{Mg}_{0} \mathrm{Zn}_{4}$ & $0.98 \pm 0.04 \mathrm{st}$ & $1.06 \pm 0.06 \mathrm{u}$ & $1.78 \pm 0.03 \mathrm{jk}$ & $1.83 \pm 0.04 \mathrm{ij}$ \\
\hline $\mathrm{N}_{20} \mathrm{P}_{50} \mathrm{~K}_{20} \mathrm{Mg}_{15} \mathrm{Zn}_{8}$ & $1.58 \pm 0.02 \mathrm{kl}$ & $1.63 \pm 0.06 \mathrm{ijk}$ & $1.85 \pm 0.02 \mathrm{ef}$ & $1.93 \pm 0.03 \mathrm{fg}$ \\
\hline $\mathrm{N}_{40} \mathrm{P}_{25} \mathrm{~K}_{20} \mathrm{Mg}_{15} \mathrm{Zn}_{0}$ & $1.27 \pm 0.01 \mathrm{qr}$ & $1.36 \pm 0.05 \mathrm{opq}$ & $1.98 \pm 0.05 \mathrm{~cd}$ & $2.17 \pm 0.07 \mathrm{~cd}$ \\
\hline $\mathrm{N}_{20} \mathrm{P}_{25} \mathrm{~K}_{0} \mathrm{Mg}_{15} \mathrm{Zn}_{0}$ & $1.97 \pm 0.06 \mathrm{a}$ & $2.16 \pm 0.04 \mathrm{a}$ & $2.32 \pm 0.12 \mathrm{a}$ & $2.45 \pm 0.03 \mathrm{ab}$ \\
\hline $\mathrm{N}_{20} \mathrm{P}_{25} \mathrm{~K}_{20} \mathrm{Mg}_{0} \mathrm{Zn}_{8}$ & $1.8 \pm 0.08 \mathrm{bc}$ & $1.71 \pm 0.02 \mathrm{fg}$ & $2.01 \pm 0.02 \mathrm{~cd}$ & $2.22 \pm 0.03 \mathrm{~cd}$ \\
\hline $\mathrm{N}_{40} \mathrm{P}_{25} \mathrm{~K}_{20} \mathrm{Mg}_{30} \mathrm{Zn}_{4}$ & $1.59 \pm 0.03 \mathrm{jk}$ & $1.62 \pm 0.03 \mathrm{ijk}$ & $2.3 \pm 0.2 \mathrm{ab}$ & $2.6 \pm 0.1 \mathrm{ab}$ \\
\hline $\mathrm{N}_{0} \mathrm{P}_{25} \mathrm{~K}_{20} \mathrm{Mg}_{30} \mathrm{Zn}_{4}$ & $1.63 \pm 0.1 \mathrm{gh}$ & $1.73 \pm 0.04 \mathrm{efg}$ & $1.8 \pm 0.03 \mathrm{gf}$ & $1.73 \pm 0.04 \mathrm{kl}$ \\
\hline $\mathrm{N}_{20} \mathrm{P}_{50} \mathrm{~K}_{40} \mathrm{Mg}_{15} \mathrm{Zn}_{4}$ & $1.86 \pm 0.07 b c$ & $2.12 \pm 0.08 \mathrm{ab}$ & $2.25 \pm 0.04 \mathrm{~b}$ & $2.42 \pm 0.09 b c$ \\
\hline $\mathrm{N}_{0} \mathrm{P}_{25} \mathrm{~K}_{20} \mathrm{Mg}_{0} \mathrm{Zn}_{4}$ & $1.2 \pm 0.02 \mathrm{qr}$ & $1.59 \pm 0.05 \mathrm{jk}$ & $1.35 \pm 0.02 \mathrm{qr}$ & $1.4 \pm 0.1 \mathrm{pqr}$ \\
\hline $\mathrm{N}_{20} \mathrm{P}_{25} \mathrm{~K}_{40} \mathrm{Mg}_{0} \mathrm{Zn}_{4}$ & $1.24 \pm 0.03 \mathrm{qr}$ & $1.37 \pm 0.05 \mathrm{opq}$ & $2.12 \pm 0.06 \mathrm{~cd}$ & $2.32 \pm 0.05 b c$ \\
\hline $\mathrm{N}_{0} \mathrm{P}_{0} \mathrm{~K}_{20} \mathrm{Mg}_{15} \mathrm{Zn}_{4}$ & $1.58 \pm 0.02 \mathrm{jk}$ & $1.62 \pm 0.03 \mathrm{ijk}$ & $1.86 \pm 0.02 \mathrm{ef}$ & $1.76 \pm 0.04 \mathrm{jk}$ \\
\hline $\mathrm{N}_{20} \mathrm{P}_{25} \mathrm{~K}_{20} \mathrm{Mg}_{30} \mathrm{Zn}_{8}$ & $1.2 \pm 0.02 \mathrm{rs}$ & $1.17 \pm 0.1 \mathrm{t}$ & $2.35 \pm 0.12 \mathrm{a}$ & $2.47 \pm 0.15 \mathrm{ab}$ \\
\hline $\mathrm{N}_{0} \mathrm{P}_{50} \mathrm{~K}_{20} \mathrm{Mg}_{15} \mathrm{Zn}_{4}$ & $1.78 \pm 0.08 \mathrm{ef}$ & $1.66 \pm 0.05 \mathrm{ghij}$ & $2.03 \pm 0.05 \mathrm{~cd}$ & $2.17 \pm 0.06 \mathrm{~cd}$ \\
\hline $\mathrm{N}_{20} \mathrm{P}_{0} \mathrm{~K}_{20} \mathrm{Mg}_{15} \mathrm{Zn}_{8}$ & $1.27 \pm 0.1 \mathrm{kl}$ & $1.40 \pm 0.07 \mathrm{nop}$ & $2.06 \pm 0.02 \mathrm{~cd}$ & $2.08 \pm 0.13 \mathrm{~cd}$ \\
\hline $\mathrm{N}_{20} \mathrm{P}_{25} \mathrm{~K}_{40} \mathrm{Mg}_{30} \mathrm{Zn}_{4}$ & $1.46 \pm 0.02 \mathrm{no}$ & $1.39 \pm 0.11$ nop & $1.76 \pm 0.03 \mathrm{mn}$ & $1.84 \pm 0.05 \mathrm{ijk}$ \\
\hline $\mathrm{N}_{0} \mathrm{P}_{25} \mathrm{~K}_{0} \mathrm{Mg}_{15} \mathrm{Zn}_{4}$ & $1.59 \pm 0.03 \mathrm{jk}$ & $1.66 \pm 0.04 \mathrm{ghij}$ & $2.15 \pm 0.02 \mathrm{~cd}$ & $2.73 \pm 0.06 \mathrm{a}$ \\
\hline $\mathrm{N}_{20} \mathrm{P}_{0} \mathrm{~K}_{0} \mathrm{Mg}_{15} \mathrm{Zn}_{4}$ & $1.69 \pm 0.1 \mathrm{hi}$ & $1.78 \pm 0.07 \mathrm{def}$ & $1.46 \pm 0.03 q$ & $1.52 \pm 0.1 \mathrm{pq}$ \\
\hline $\mathrm{N}_{40} \mathrm{P}_{25} \mathrm{~K}_{20} \mathrm{Mg}_{15} \mathrm{Zn}_{8}$ & $1.37 \pm 0.07 \mathrm{op}$ & $1.45 \pm 0.07 \mathrm{mn}$ & $1.59 \pm 0.02 \mathrm{op}$ & $1.68 \pm 0.02 \mathrm{klm}$ \\
\hline $\mathrm{N}_{40} \mathrm{P}_{25} \mathrm{~K}_{40} \mathrm{Mg}_{15} \mathrm{Zn}_{4}$ & $1.68 \pm 0.05 \mathrm{hi}$ & $1.71 \pm 0.06 \mathrm{fgh}$ & $1.89 \pm 0.06 \mathrm{de}$ & $1.95 \pm 0.05 \mathrm{fg}$ \\
\hline $\mathrm{N}_{20} \mathrm{P}_{50} \mathrm{~K}_{20} \mathrm{Mg}_{30} \mathrm{Zn}_{4}$ & $1.57 \pm 0.01 \mathrm{kl}$ & $1.66 \pm 0.01 \mathrm{ghi}$ & $1.49 \pm 0.03 q$ & $1.59 \pm 0.09 \mathrm{pq}$ \\
\hline $\mathrm{N}_{20} \mathrm{P}_{0} \mathrm{~K}_{40} \mathrm{Mg}_{15} \mathrm{Zn}_{4}$ & $1.68 \pm 0.02 \mathrm{ij}$ & $1.83 \pm 0.03 \mathrm{~cd}$ & $2.05 \pm 0.04 \mathrm{~d}$ & $2.26 \pm 0.09 \mathrm{bcd}$ \\
\hline $\mathrm{N}_{20} \mathrm{P}_{25} \mathrm{~K}_{40} \mathrm{Mg}_{15} \mathrm{Zn}_{8}$ & $1.42 \pm 0.03 \mathrm{no}$ & $1.39 \pm 0.03 \mathrm{nop}$ & $1.76 \pm 0.09 \mathrm{mn}$ & $1.71 \pm 0.06 \mathrm{op}$ \\
\hline $\mathrm{N}_{20} \mathrm{P}_{25} \mathrm{~K}_{0} \mathrm{Mg}_{15} \mathrm{Zn}_{8}$ & $1.56 \pm 0.03 \mathrm{kl}$ & $1.68 \pm 0.03 \mathrm{ghi}$ & $1.2 \pm 0.03 \mathrm{r}$ & $1.3 \pm 0.05 \mathrm{qr}$ \\
\hline $\mathrm{N}_{0} \mathrm{P}_{25} \mathrm{~K}_{40} \mathrm{Mg}_{15} \mathrm{Zn}_{4}$ & $1.69 \pm 0.02 \mathrm{hi}$ & $1.56 \pm 0.1 \mathrm{kl}$ & $2.01 \pm 0.04 \mathrm{~cd}$ & $2.03 \pm 0.06 \mathrm{ef}$ \\
\hline $\mathrm{N}_{20} \mathrm{P}_{25} \mathrm{~K}_{0} \mathrm{Mg}_{0} \mathrm{Zn}_{4}$ & $1.23 \pm 0.02 \mathrm{rs}$ & $0.77 \pm 0.09 \mathrm{vw}$ & $1.48 \pm 0.02 \mathrm{op}$ & $1.55 \pm 0.07 \mathrm{mn}$ \\
\hline $\mathrm{N}_{20} \mathrm{P}_{0} \mathrm{~K}_{20} \mathrm{Mg}_{30} \mathrm{Zn}_{4}$ & $1.2 \pm 0.03 \mathrm{mn}$ & $1.31 \pm 0.03 \mathrm{qrs}$ & $1.98 \pm 0.02 \mathrm{de}$ & $2.15 \pm 0.02 \mathrm{~cd}$ \\
\hline $\mathrm{N}_{20} \mathrm{P}_{25} \mathrm{~K}_{20} \mathrm{Mg}_{15} \mathrm{Zn}_{4}$ & $1.78 \pm 0.04 \mathrm{efg}$ & $1.43 \pm 0.03 \mathrm{no}$ & $2.12 \pm 0.03 \mathrm{bc}$ & $1.73 \pm 0.09$ op \\
\hline $\mathrm{N}_{0} \mathrm{P}_{25} \mathrm{~K}_{20} \mathrm{Mg}_{15} \mathrm{Zn}_{0}$ & $1.43 \pm 0.03 \mathrm{mn}$ & $1.04 \pm 0.03 \mathrm{u}$ & $1.2 \pm 0.02 \mathrm{r}$ & $1.1 \pm 0.02 \mathrm{r}$ \\
\hline $\mathrm{N}_{20} \mathrm{P}_{25} \mathrm{~K}_{20} \mathrm{Mg}_{30} \mathrm{Zn}_{0}$ & $1.68 \pm 0.08 \mathrm{fgh}$ & $1.56 \pm 0.09 \mathrm{kl}$ & $1.69 \pm 0.03 \mathrm{mn}$ & $1.77 \pm 0.04 \mathrm{no}$ \\
\hline $\mathrm{N}_{20} \mathrm{P}_{25} \mathrm{~K}_{40} \mathrm{Mg}_{15} \mathrm{Zn}_{0}$ & $1.25 \pm 0.03 \mathrm{kl}$ & $1.06 \pm 0.02 \mathrm{u}$ & $1.76 \pm 0.02 \mathrm{kl}$ & $1.77 \pm 0.15 \mathrm{jkl}$ \\
\hline $\mathrm{N}_{20} \mathrm{P}_{50} \mathrm{~K}_{20} \mathrm{Mg}_{0} \mathrm{Zn}_{4}$ & $1.16 \pm 0.06 \mathrm{mn}$ & $1.28 \pm 0.05 \mathrm{rs}$ & $2.13 \pm 0.02 b$ & $2.29 \pm 0.3 \mathrm{bcd}$ \\
\hline $\mathrm{N}_{40} \mathrm{P}_{0} \mathrm{~K}_{20} \mathrm{Mg}_{15} \mathrm{Zn}_{4}$ & $1.23 \pm 0.03 \mathrm{mn}$ & $1.42 \pm 0.07 \mathrm{no}$ & $2.16 \pm 0.06 \mathrm{~b}$ & $2.10 \pm 0.04 \mathrm{ef}$ \\
\hline $\mathrm{N}_{20} \mathrm{P}_{25} \mathrm{~K}_{20} \mathrm{Mg}_{15} \mathrm{Zn}_{4}$ & $1.86 \pm 0.02 b c$ & $1.63 \pm 0.02 \mathrm{ijk}$ & $1.74 \pm 0.05 \mathrm{kl}$ & $1.77 \pm 0.05 \mathrm{jklm}$ \\
\hline $\mathrm{N}_{20} \mathrm{P}_{25} \mathrm{~K}_{20} \mathrm{Mg}_{15} \mathrm{Zn}_{4}$ & $1.69 \pm 0.05 \mathrm{gh}$ & $1.06 \pm 0.02 \mathrm{u}$ & $1.86 \pm 0.02 \mathrm{de}$ & $2.27 \pm 0.01 \mathrm{bcde}$ \\
\hline $\mathrm{N}_{20} \mathrm{P}_{25} \mathrm{~K}_{20} \mathrm{Mg}_{15} \mathrm{Zn}_{4}$ & $1.59 \pm 0.1 \mathrm{ij}$ & $1.32 \pm 0.04 \mathrm{pqr}$ & $1.26 \pm 0.05 \mathrm{r}$ & $1.32 \pm 0.08 \mathrm{qr}$ \\
\hline $\mathrm{N}_{20} \mathrm{P}_{25} \mathrm{~K}_{20} \mathrm{Mg}_{0} \mathrm{Zn}_{0}$ & $1.43 \pm 0.02 \mathrm{mn}$ & $1.24 \pm 0.09 \mathrm{st}$ & $1.8 \pm 0.09 \mathrm{de}$ & $1.9 \pm 0.1 \mathrm{gh}$ \\
\hline $\mathrm{N}_{0} \mathrm{P}_{25} \mathrm{~K}_{20} \mathrm{Mg}_{15} \mathrm{Zn}_{8}$ & $1.73 \pm 0.02 \mathrm{ef}$ & $1.82 \pm 0.04 \mathrm{cde}$ & $1.52 \pm 0.1 \mathrm{pq}$ & $1.44 \pm 0.03 \mathrm{pq}$ \\
\hline $\mathrm{N}_{20} \mathrm{P}_{0} \mathrm{~K}_{20} \mathrm{Mg}_{15} \mathrm{Zn}_{0}$ & $1.56 \pm 0.01 \mathrm{gh}$ & $1.62 \pm 0.01 \mathrm{ijk}$ & $1.78 \pm 0.02 \mathrm{mn}$ & $1.87 \pm 0.02 \mathrm{hi}$ \\
\hline $\mathrm{N}_{20} \mathrm{P}_{50} \mathrm{~K}_{0} \mathrm{Mg}_{15} \mathrm{Zn}_{4}$ & $1.56 \pm 0.05 \mathrm{hi}$ & $1.73 \pm 0.01 \mathrm{fg}$ & $2.13 \pm 0.1 b$ & $2.23 \pm 0.06 \mathrm{bcd}$ \\
\hline $\mathrm{N}_{40} \mathrm{P}_{25} \mathrm{~K}_{0} \mathrm{Mg}_{15} \mathrm{Zn}_{4}$ & $1.79 \pm 0.04 \mathrm{ef}$ & $2.06 \pm 0.02 b$ & $2.32 \pm 0.15 \mathrm{a}$ & $2.43 \pm 0.08 \mathrm{ab}$ \\
\hline
\end{tabular}




\section{Continued}

\begin{tabular}{ccccc}
\hline $\mathrm{N}_{40} \mathrm{P}_{50} \mathrm{~K}_{20} \mathrm{Mg}_{15} \mathrm{Zn}_{4}$ & $1.69 \pm 0.03 \mathrm{gh}$ & $1.89 \pm 0.07 \mathrm{c}$ & $2.10 \pm 0.03 \mathrm{~b}$ & $2.19 \pm 0.03 \mathrm{cde}$ \\
$\mathrm{N}_{20} \mathrm{P}_{25} \mathrm{~K}_{20} \mathrm{Mg}_{15} \mathrm{Zn}_{4}$ & $1.98 \pm 0.04 \mathrm{a}$ & $1.84 \pm 0.02 \mathrm{~cd}$ & $2.13 \pm 0.02 \mathrm{~b}$ & $1.97 \pm 0.03 \mathrm{fgh}$ \\
$\mathrm{N}_{20} \mathrm{P}_{25} \mathrm{~K}_{0} \mathrm{Mg}_{30} \mathrm{Zn}_{4}$ & $1.75 \pm 0.06 \mathrm{efg}$ & $1.66 \pm 0.03 \mathrm{hij}$ & $1.99 \pm 0.03 \mathrm{~cd}$ & $2.05 \pm 0.05 \mathrm{de}$ \\
$\mathrm{N}_{20} \mathrm{P}_{25} \mathrm{~K}_{20} \mathrm{Mg}_{15} \mathrm{Zn}_{4}$ & $1.89 \pm 0.03 \mathrm{bc}$ & $1.50 \pm 0.05 \mathrm{~lm}$ & $1.78 \pm 0.03 \mathrm{mn}$ & $1.83 \pm 0.02 \mathrm{ij}$ \\
$\mathrm{N}_{40} \mathrm{P}_{25} \mathrm{~K}_{20} \mathrm{Mg}_{0} \mathrm{Zn}_{4}$ & $0.89 \pm 0.02 \mathrm{r}$ & $0.75 \pm 0.01 \mathrm{vw}$ & $1.02 \pm 0.01 \mathrm{q}$ & $1.06 \pm 0.01 \mathrm{r}$ \\
$\mathrm{N}_{20} \mathrm{P}_{50} \mathrm{~K}_{20} \mathrm{Mg}_{15} \mathrm{Zn}_{0}$ & $0.78 \pm 0.03 \mathrm{rs}$ & $0.81 \pm 0.04 \mathrm{v}$ & $1.06 \pm 0.05 \mathrm{q}$ & $1.09 \pm 0.07 \mathrm{r}$ \\
\hline
\end{tabular}

In a column, means followed by the same alphabetic letters are not significantly different $(p>0.05)$ according to Student Newman-Keuls test.

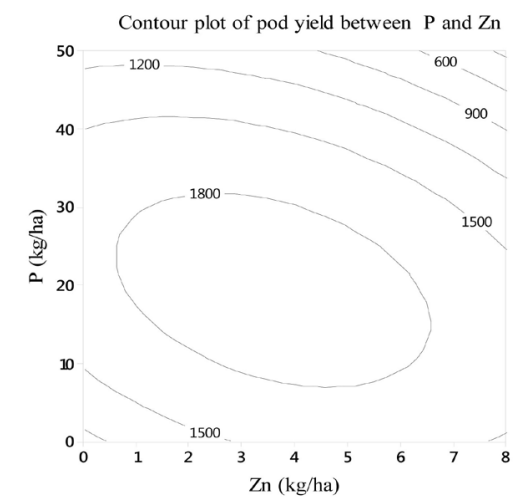

(a)



(c)

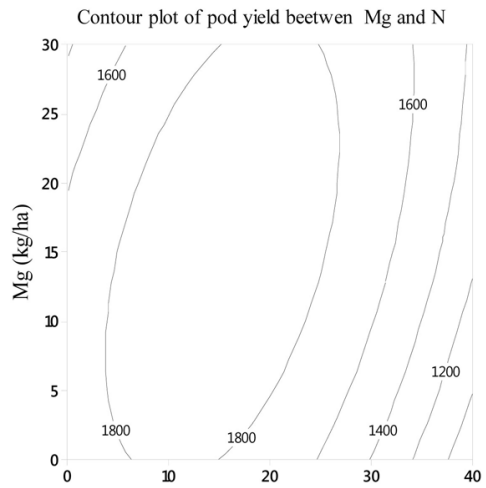

(e)

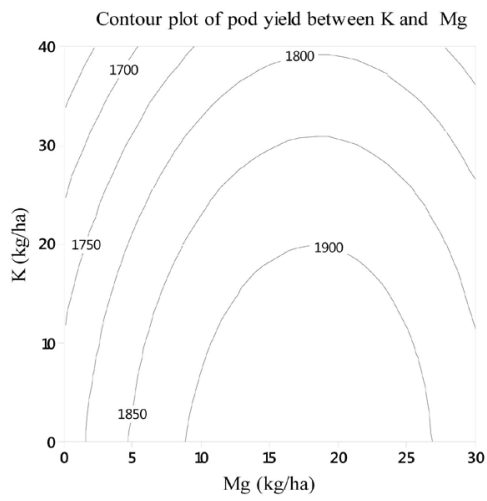

(b)

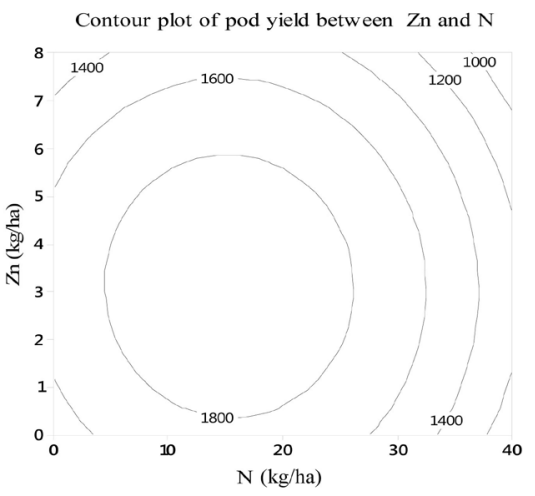

(d)



(f)

Figure 2. Response surface plots of groundnut pod yields regarding the types and rates of nutrients $\mathrm{N}, \mathrm{P}, \mathrm{K}, \mathrm{Mg}$ and $\mathrm{Zn}$ applied. 
Table 3. Average values ( \pm standard errors) of aboveground biomass and pod yields of groundnut regarding the combined doses of N, P, K, Mg and Zn during 2018 and 2019 growing seasons in the site of North-Benin.

\begin{tabular}{|c|c|c|c|c|}
\hline \multirow{2}{*}{ Treatments } & \multicolumn{2}{|c|}{ Pod yields $\left(\mathrm{t} \cdot \mathrm{DM} \cdot \mathrm{ha}^{-1}\right)$} & \multicolumn{2}{|c|}{ Aboveground biomass $\left(\mathrm{t} \cdot \mathrm{DM} \cdot \mathrm{ha}^{-1}\right)$} \\
\hline & 2018 & 2019 & 2018 & 2019 \\
\hline $\mathrm{N}_{0} \mathrm{P}_{0} \mathrm{~K}_{0} \mathrm{Mg}_{0} \mathrm{Zn}_{0}$ & $0.63 \pm 0.03 p$ & $0.61 \pm 0.05 z$ & $0.67 \pm 0.01 \mathrm{~m}$ & $0.75 \pm 0.02 \mathrm{~m}$ \\
\hline $\mathrm{N}_{20} \mathrm{P}_{0} \mathrm{~K}_{20} \mathrm{Mg}_{0} \mathrm{Zn}_{4}$ & $1.36 \pm 0.01 \mathrm{kl}$ & $1.63 \pm 0.03 \mathrm{jklm}$ & $1.11 \pm 0.02 \mathrm{kl}$ & $1.73 \pm 0.02 \mathrm{efg}$ \\
\hline $\mathrm{N}_{20} \mathrm{P}_{50} \mathrm{~K}_{20} \mathrm{Mg}_{15} \mathrm{Zn}_{8}$ & $0.52 \pm 0.02 p$ & $1.31 \pm 0.08 \mathrm{u}$ & $1.92 \pm 0.03 \mathrm{ab}$ & $1.86 \pm 0.03 \mathrm{bcd}$ \\
\hline $\mathrm{N}_{40} \mathrm{P}_{25} \mathrm{~K}_{20} \mathrm{Mg}_{15} \mathrm{Zn}_{0}$ & $1.47 \pm 0.02 \mathrm{hijk}$ & $1.47 \pm 0.03 \mathrm{qrs}$ & $2.01 \pm 0.1 \mathrm{abcd}$ & $1.89 \pm 0.03 \mathrm{bcd}$ \\
\hline $\mathrm{N}_{20} \mathrm{P}_{25} \mathrm{~K}_{0} \mathrm{Mg}_{15} \mathrm{Zn}_{0}$ & $1.79 \pm 0.02 \mathrm{cb}$ & $1.95 \pm 0.02 \mathrm{ab}$ & $1.66 \pm 0.12 \mathrm{defgh}$ & $1.89 \pm 0.02 \mathrm{bcd}$ \\
\hline $\mathrm{N}_{20} \mathrm{P}_{25} \mathrm{~K}_{20} \mathrm{Mg}_{0} \mathrm{Zn}_{8}$ & $1.59 \pm 0.04 \mathrm{ghi}$ & $1.53 \pm 0.07 \mathrm{opqr}$ & $1.37 \pm 0.04 \mathrm{kl}$ & $1.45 \pm 0.03 \mathrm{ij}$ \\
\hline $\mathrm{N}_{40} \mathrm{P}_{25} \mathrm{~K}_{20} \mathrm{Mg}_{30} \mathrm{Zn}_{4}$ & $1.49 \pm 0.03 \mathrm{hijk}$ & $1.58 \pm 0.03 p q$ & $2.19 \pm 0.18 \mathrm{a}$ & $2.01 \pm 0.11 \mathrm{abc}$ \\
\hline $\mathrm{N}_{0} \mathrm{P}_{25} \mathrm{~K}_{20} \mathrm{Mg}_{30} \mathrm{Zn}_{4}$ & $1.47 \pm 0.05 \mathrm{hijk}$ & $1.54 \pm 0.06 \mathrm{opqr}$ & $0.96 \pm 0.1 \mathrm{~m}$ & $1.2 \pm 0.02 \mathrm{kl}$ \\
\hline $\mathrm{N}_{20} \mathrm{P}_{50} \mathrm{~K}_{40} \mathrm{Mg}_{15} \mathrm{Zn}_{4}$ & $1.8 \pm 0.01 b$ & $1.88 \pm 0.02 \mathrm{abc}$ & $1.39 \pm 0.01 \mathrm{kl}$ & $1.59 \pm 0.02 \mathrm{kl}$ \\
\hline $\mathrm{N}_{0} \mathrm{P}_{25} \mathrm{~K}_{20} \mathrm{Mg}_{0} \mathrm{Zn}_{4}$ & $1.56 \pm 0.02$ efghi & $1.71 \pm 0.04$ fghi & $0.88 \pm 0.01 \mathrm{~lm}$ & $1.02 \pm 0.03 \mathrm{kl}$ \\
\hline $\mathrm{N}_{20} \mathrm{P}_{25} \mathrm{~K}_{40} \mathrm{Mg}_{0} \mathrm{Zn}_{4}$ & $1.78 \pm 0.03 \mathrm{cb}$ & $1.93 \pm 0.2 \mathrm{abc}$ & $1.54 \pm 0.07 \mathrm{k}$ & $1.87 \pm 0.02 \mathrm{~cd}$ \\
\hline $\mathrm{N}_{0} \mathrm{P}_{0} \mathrm{~K}_{20} \mathrm{Mg}_{15} \mathrm{Zn}_{4}$ & $1.74 \pm 0.01 \mathrm{cbd}$ & $1.76 \pm 0.2 \mathrm{ghij}$ & $1.69 \pm 0.03 \mathrm{efgh}$ & $1.58 \pm 0.11 \mathrm{kl}$ \\
\hline $\mathrm{N}_{20} \mathrm{P}_{25} \mathrm{~K}_{20} \mathrm{Mg}_{30} \mathrm{Zn}_{8}$ & $1.74 \pm 0.01 \mathrm{cbd}$ & $1.75 \pm 0.03 \mathrm{gh}$ & $1.27 \pm 0.03 \mathrm{kl}$ & $1.39 \pm 0.02 \mathrm{~m}$ \\
\hline $\mathrm{N}_{0} \mathrm{P}_{50} \mathrm{~K}_{20} \mathrm{Mg}_{15} \mathrm{Zn}_{4}$ & $0.65 \pm 0.01$ op & $1.50 \pm 0.03 \mathrm{pqr}$ & $1.33 \pm 0.04 \mathrm{kl}$ & $1.45 \pm 0.2 \mathrm{jk}$ \\
\hline $\mathrm{N}_{20} \mathrm{P}_{0} \mathrm{~K}_{20} \mathrm{Mg}_{15} \mathrm{Zn}_{8}$ & $1.61 \pm 0.03 \mathrm{efgh}$ & $1.64 \pm 0.01 \mathrm{lmn}$ & $1.32 \pm 0.06 \mathrm{kl}$ & $1.58 \pm 0.03 \mathrm{jk}$ \\
\hline $\mathrm{N}_{20} \mathrm{P}_{25} \mathrm{~K}_{40} \mathrm{Mg}_{30} \mathrm{Zn}_{4}$ & $1.70 \pm 0.02 \mathrm{cbd}$ & $1.73 \pm 0.02 \mathrm{hi}$ & $1.55 \pm 0.01 \mathrm{i}$ & $1.65 \pm 0.11 \mathrm{hij}$ \\
\hline $\mathrm{N}_{0} \mathrm{P}_{25} \mathrm{~K}_{0} \mathrm{Mg}_{15} \mathrm{Zn}_{4}$ & $1.55 \pm 0.03$ efghi & $1.60 \pm 0.03 \mathrm{nop}$ & $1.26 \pm 0.05 \mathrm{kl}$ & $1.58 \pm 0.00 \mathrm{kl}$ \\
\hline $\mathrm{N}_{20} \mathrm{P}_{0} \mathrm{~K}_{0} \mathrm{Mg}_{15} \mathrm{Zn}_{4}$ & $1.27 \pm 0.001$ & $1.37 \pm 0.03 \mathrm{stu}$ & $1.16 \pm 0.05 \mathrm{kl}$ & $1.29 \pm 0.02 \mathrm{mn}$ \\
\hline $\mathrm{N}_{40} \mathrm{P}_{25} \mathrm{~K}_{20} \mathrm{Mg}_{15} \mathrm{Zn}_{8}$ & $1.63 \pm 0.01 \mathrm{efgh}$ & $1.85 \pm 0.02 \mathrm{cdef}$ & $2.03 \pm 0.02 \mathrm{abcd}$ & $1.98 \pm 0.02 \mathrm{ab}$ \\
\hline $\mathrm{N}_{40} \mathrm{P}_{25} \mathrm{~K}_{40} \mathrm{Mg}_{15} \mathrm{Zn}_{4}$ & $0.62 \pm 0.01 p$ & $0.85 \pm 0.03 x$ & $2.18 \pm 0.04 \mathrm{ab}$ & $2.2 \pm 0.03 \mathrm{a}$ \\
\hline $\mathrm{N}_{20} \mathrm{P}_{50} \mathrm{~K}_{20} \mathrm{Mg}_{30} \mathrm{Zn}_{4}$ & $0.76 \pm 0.03$ on & $1.21 \pm 0.01 \mathrm{v}$ & $1.62 \pm 0.02 \mathrm{ghi}$ & $1.78 \pm 0.02 \mathrm{~cd}$ \\
\hline $\mathrm{N}_{20} \mathrm{P}_{0} \mathrm{~K}_{40} \mathrm{Mg}_{15} \mathrm{Zn}_{4}$ & $1.71 \pm 0.00 \mathrm{bcde}$ & $1.83 \pm 0.1 \mathrm{cdefg}$ & $1.77 \pm 0.01 \mathrm{defg}$ & $1.86 \pm 0.02 \mathrm{bcd}$ \\
\hline $\mathrm{N}_{20} \mathrm{P}_{25} \mathrm{~K}_{40} \mathrm{Mg}_{15} \mathrm{Zn}_{8}$ & $1.51 \pm 0.01$ fghij & $1.81 \pm 0.01 \mathrm{~cd}$ & $1.29 \pm 0.02 \mathrm{kl}$ & $1.37 \pm 0.02 \mathrm{kl}$ \\
\hline $\mathrm{N}_{20} \mathrm{P}_{25} \mathrm{~K}_{0} \mathrm{Mg}_{15} \mathrm{Zn}_{8}$ & $1.40 \pm 0.02 \mathrm{jk}$ & $1.44 \pm 0.02 \mathrm{rst}$ & $0.94 \pm 0.01 \mathrm{~m}$ & $1.05 \pm 0.02 \mathrm{~m}$ \\
\hline $\mathrm{N}_{0} \mathrm{P}_{25} \mathrm{~K}_{40} \mathrm{Mg}_{15} \mathrm{Zn}_{4}$ & $1.65 \pm 0.01 \mathrm{fg}$ & $1.70 \pm 0.01 \mathrm{hij}$ & $1.35 \pm 0.12 \mathrm{kl}$ & $1.46 \pm 0.1 \mathrm{gh}$ \\
\hline $\mathrm{N}_{20} \mathrm{P}_{25} \mathrm{~K}_{0} \mathrm{Mg}_{0} \mathrm{Zn}_{4}$ & $1.68 \pm 0.07 \mathrm{bcde}$ & $1.56 \pm 0.00 \mathrm{opqr}$ & $1.22 \pm 0.16 \mathrm{kl}$ & $1.35 \pm 0.05 \mathrm{kl}$ \\
\hline $\mathrm{N}_{20} \mathrm{P}_{0} \mathrm{~K}_{20} \mathrm{Mg}_{30} \mathrm{Zn}_{4}$ & $1.69 \pm 0.0 \mathrm{bcde}$ & $1.75 \pm 0.02 \mathrm{efg}$ & $1.32 \pm 0.02 \mathrm{kl}$ & $1.48 \pm 0.03 \mathrm{kl}$ \\
\hline $\mathrm{N}_{20} \mathrm{P}_{25} \mathrm{~K}_{20} \mathrm{Mg}_{15} \mathrm{Zn}_{4}$ & $1.94 \pm 0.01 \mathrm{a}$ & $1.97 \pm 0.02 \mathrm{a}$ & $1.36 \pm 0.11 \mathrm{ijkl}$ & $1.78 \pm 0.01 \mathrm{~cd}$ \\
\hline $\mathrm{N}_{0} \mathrm{P}_{25} \mathrm{~K}_{20} \mathrm{Mg}_{15} \mathrm{Zn}_{0}$ & $1.62 \pm 0.01 \mathrm{gh}$ & $1.66 \pm 0.01 \mathrm{klm}$ & $1.65 \pm 0.01 \mathrm{ghi}$ & $1.78 \pm 0.02 \mathrm{~cd}$ \\
\hline $\mathrm{N}_{20} \mathrm{P}_{25} \mathrm{~K}_{20} \mathrm{Mg}_{30} \mathrm{Zn}_{0}$ & $1.63 \pm 0.04 \mathrm{gh}$ & $1.64 \pm 0.02 \mathrm{jklm}$ & $1.55 \pm 0.06 \mathrm{k}$ & $1.69 \pm 0.03 \mathrm{fg}$ \\
\hline $\mathrm{N}_{20} \mathrm{P}_{25} \mathrm{~K}_{40} \mathrm{Mg}_{15} \mathrm{Zn}_{0}$ & $1.65 \pm 0.03 \mathrm{fg}$ & $1.80 \pm 0.04 \mathrm{~cd}$ & $1.52 \pm 0.11 \mathrm{ijk}$ & $1.68 \pm 0.1 \mathrm{ghi}$ \\
\hline $\mathrm{N}_{20} \mathrm{P}_{50} \mathrm{~K}_{20} \mathrm{Mg}_{0} \mathrm{Zn}_{4}$ & $0.76 \pm 0.02 \mathrm{no}$ & $0.83 \pm 0.01 \mathrm{x}$ & $1.43 \pm 0.05 \mathrm{kl}$ & $1.64 \pm 0.2 \mathrm{hi}$ \\
\hline $\mathrm{N}_{40} \mathrm{P}_{0} \mathrm{~K}_{20} \mathrm{Mg}_{15} \mathrm{Zn}_{4}$ & $0.92 \pm 0.08 \mathrm{~m}$ & $1.35 \pm 0.07 \mathrm{tu}$ & $2.18 \pm 0.18 \mathrm{ab}$ & $2 \pm 0.03 \mathrm{ab}$ \\
\hline $\mathrm{N}_{20} \mathrm{P}_{25} \mathrm{~K}_{20} \mathrm{Mg}_{15} \mathrm{Zn}_{4}$ & $1.80 \pm 0.06 \mathrm{~b}$ & $1.89 \pm 0.02 \mathrm{ab}$ & $1.31 \pm 0.09 \mathrm{kl}$ & $1.42 \pm 0.2 \mathrm{hij}$ \\
\hline $\mathrm{N}_{20} \mathrm{P}_{25} \mathrm{~K}_{20} \mathrm{Mg}_{15} \mathrm{Zn}_{4}$ & $1.58 \pm 0.04$ fghi & $1.67 \pm 0.01 \mathrm{hij}$ & $1.43 \pm 0.00 \mathrm{hij}$ & $1.58 \pm 0.03 \mathrm{gh}$ \\
\hline $\mathrm{N}_{20} \mathrm{P}_{25} \mathrm{~K}_{20} \mathrm{Mg}_{15} \mathrm{Zn}_{4}$ & $1.64 \pm 0.06 \mathrm{fgh}$ & $1.80 \pm 0.02 \mathrm{cde}$ & $1.34 \pm 0.01 \mathrm{kl}$ & $1.69 \pm 0.01 \mathrm{def}$ \\
\hline
\end{tabular}




\section{Continued}

\begin{tabular}{ccccc}
\hline $\mathrm{N}_{20} \mathrm{P}_{25} \mathrm{~K}_{20} \mathrm{Mg}_{0} \mathrm{Zn}_{0}$ & $1.68 \pm 0.01 \mathrm{bcde}$ & $1.81 \pm 0.03 \mathrm{cde}$ & $1.34 \pm 0.1 \mathrm{kl}$ & $1.75 \pm 0.2 \mathrm{~cd}$ \\
$\mathrm{~N}_{0} \mathrm{P}_{25} \mathrm{~K}_{20} \mathrm{Mg}_{15} \mathrm{Zn}_{8}$ & $1.72 \pm 0.00 \mathrm{cbde}$ & $1.83 \pm 0.00 \mathrm{bcd}$ & $0.94 \pm 0.07 \mathrm{~lm}$ & $1.23 \pm 0.2 \mathrm{kl}$ \\
$\mathrm{N}_{20} \mathrm{P}_{0} \mathrm{~K}_{20} \mathrm{Mg}_{15} \mathrm{Zn}_{0}$ & $1.64 \pm 0.01 \mathrm{efgh}$ & $1.58 \pm 0.01 \mathrm{pq}$ & $1.45 \pm 0.15 \mathrm{ijk}$ & $1.68 \pm 0.3 \mathrm{de}$ \\
$\mathrm{N}_{20} \mathrm{P}_{50} \mathrm{~K}_{0} \mathrm{Mg}_{15} \mathrm{Zn}_{4}$ & $1.44 \pm 0.02 \mathrm{ijk}$ & $1.55 \pm 0.03 \mathrm{qr}$ & $1.51 \pm 0.12 \mathrm{hij}$ & $1.79 \pm 0.01 \mathrm{~cd}$ \\
$\mathrm{~N}_{40} \mathrm{P}_{25} \mathrm{~K}_{0} \mathrm{Mg}_{15} \mathrm{Zn}_{4}$ & $1.66 \pm 0.02 \mathrm{ef}$ & $1.6 \pm 0.01 \mathrm{klm}$ & $2.16 \pm 0.12 \mathrm{ab}$ & $2.3 \pm 0.02 \mathrm{a}$ \\
$\mathrm{N}_{40} \mathrm{P}_{50} \mathrm{~K}_{20} \mathrm{Mg}_{15} \mathrm{Zn}_{4}$ & $0.55 \pm 0.01 \mathrm{p}$ & $1.03 \pm 0.0 \mathrm{w}$ & $2.17 \pm 0.21 \mathrm{ab}$ & $2.1 \pm 0.01 \mathrm{ab}$ \\
$\mathrm{N}_{20} \mathrm{P}_{25} \mathrm{~K}_{20} \mathrm{Mg}_{15} \mathrm{Zn}_{4}$ & $1.62 \pm 0.1 \mathrm{fgh}$ & $1.68 \pm 0.02 \mathrm{hijk}$ & $1.48 \pm 0.1 \mathrm{ijk}$ & $1.57 \pm 0.02 \mathrm{gh}$ \\
$\mathrm{N}_{20} \mathrm{P}_{25} \mathrm{~K}_{0} \mathrm{Mg}_{30} \mathrm{Zn}_{4}$ & $1.59 \pm 0.02 \mathrm{fghi}$ & $1.62 \pm 0.03 \mathrm{jklm}$ & $1.81 \pm 0.1 \mathrm{cdef}$ & $1.86 \pm 0.02 \mathrm{~cd}$ \\
$\mathrm{~N}_{20} \mathrm{P}_{25} \mathrm{~K}_{20} \mathrm{Mg}_{15} \mathrm{Zn}_{4}$ & $1.63 \pm 0.01 \mathrm{fgh}$ & $1.69 \pm 0.04 \mathrm{ghij}$ & $1.24 \pm 0.1 \mathrm{kl}$ & $1.35 \pm 0.03 \mathrm{kl}$ \\
$\mathrm{N}_{40} \mathrm{P}_{25} \mathrm{~K}_{20} \mathrm{Mg}_{0} \mathrm{Zn}_{4}$ & $0.57 \pm 0.04 \mathrm{p}$ & $0.72 \pm 0.03 \mathrm{y}$ & $2.12 \pm 0.2 \mathrm{abc}$ & $1.9 \pm 0.2 \mathrm{ab}$ \\
$\mathrm{N}_{20} \mathrm{P}_{50} \mathrm{~K}_{20} \mathrm{Mg}_{15} \mathrm{Zn}_{0}$ & $0.78 \pm 0.01 \mathrm{n}$ & $0.77 \pm 0.01 \mathrm{xy}$ & $1.68 \pm 0.1 \mathrm{efgh}$ & $1.48 \pm 0.2 \mathrm{gh}$ \\
\hline
\end{tabular}

In a column, means followed by the same alphabetic letters are not significantly different $(p>0.05)$ according to Student Newman-Keuls test.

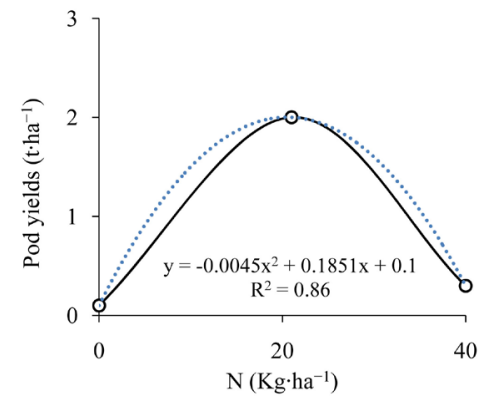

(a)

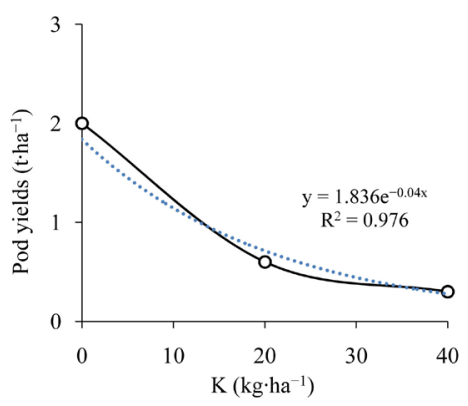

(c)

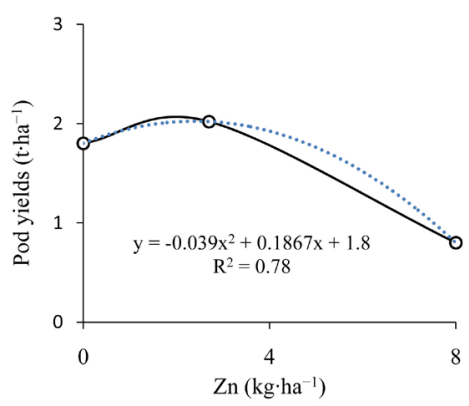

(e)

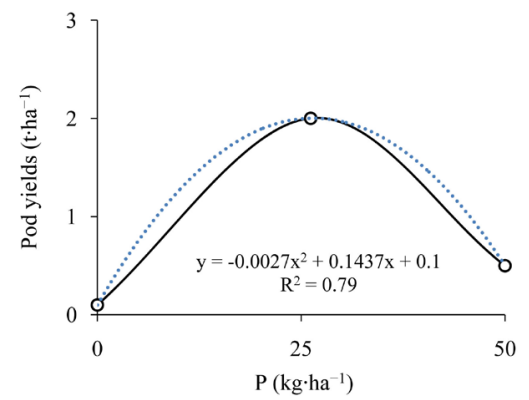

(b)

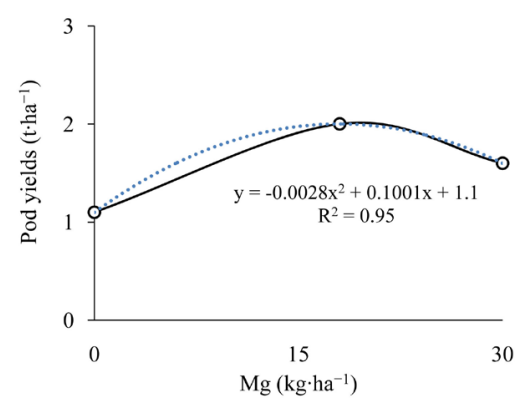

(d)

Figure 3. Response curve plots of groundnut pod yields regarding the type and rates of nutrients $\mathrm{N}, \mathrm{P}, \mathrm{K}, \mathrm{Mg}$ and $\mathrm{Zn}$ applied in the site of the north Benin. 


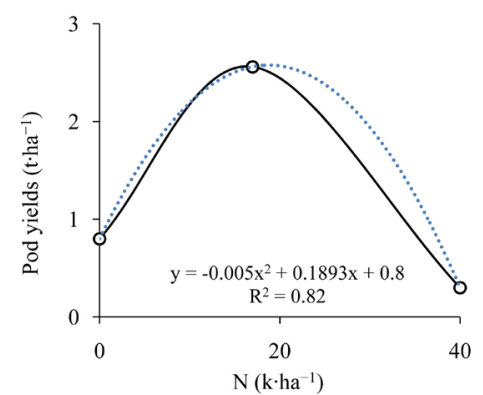

(a)



(c)

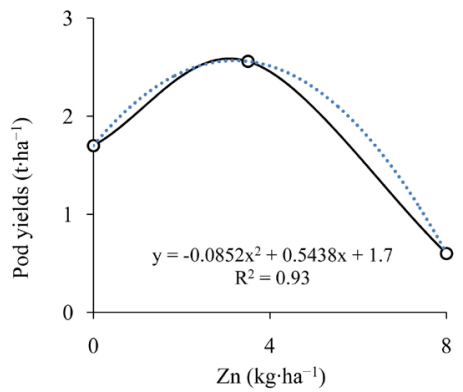

(e)

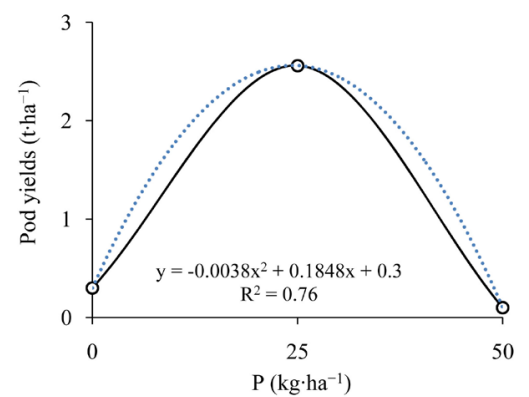

(b)

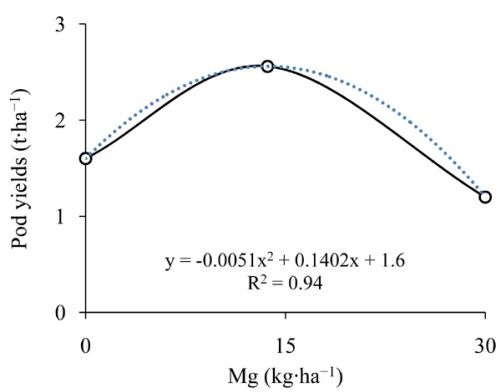

(d)

Figure 4. Response curve plots of groundnut pod yields regarding the types and rates of nutrients $\mathrm{N}, \mathrm{P}, \mathrm{K}, \mathrm{Mg}$ and $\mathrm{Zn}$ applied in the site of Centre Benin.

\subsection{Determination of the Optimal Doses of Each Type and Rate of Nutrients N, P, K, Mg and Zn Applied}

Analysis of the response surface results showed that quadratic model (at the site of Centre Benin) and quadratic with interaction (at the site of north Benin) were highly significant ( $\mathrm{p}=0.000, \mathrm{R}^{2}=0.98$ ) estimating groundnut pod yields versus variation of $\mathrm{N}, \mathrm{P}, \mathrm{K}, \mathrm{Mg}$ and $\mathrm{Zn}$ doses. The regression equations (Equation (1) for the site of Centre and Equation (2) for the site of North Benin) are:

Equation (1): Pod yield $\left(\mathrm{kg} \cdot \mathrm{ha}^{-1}\right)=506+55.2 \mathrm{~N}+62.5 \mathrm{P}+11 \mathrm{~K}+40.2 \mathrm{Mg}+$ $126.6 \mathrm{Zn}-1.6 \mathrm{~N}^{2}-1.3 \mathrm{P}^{2}-0.7 \mathrm{~K}^{2}-1.5 \mathrm{Mg}^{2}-20.2 \mathrm{Zn}^{2}$

Equation (2): Pod yield $\left(\mathrm{kg} \cdot \mathrm{ha}^{-1}\right)=597+25.6 \mathrm{~N}+59.1 \mathrm{P}+20 \mathrm{~K}+14.1 \mathrm{Mg}+$ $167.5 \mathrm{Zn}-1.12 \mathrm{~N}^{2}-1.01 \mathrm{P}^{2}-0.08 \mathrm{~K}^{2}-0.53 \mathrm{Mg}^{2}-17.4 \mathrm{Zn}^{2}+0.37 \mathrm{~N} * \mathrm{P}-0.53 \mathrm{~N} *$ $\mathrm{K}+0.7 \mathrm{~N} * \mathrm{Mg}-0.14 \mathrm{~N} * \mathrm{Zn}-0.56 \mathrm{P} * \mathrm{~K}-0.36 \mathrm{P} * \mathrm{Mg}-3 \mathrm{P} * \mathrm{Zn}+0.02 \mathrm{~K} * \mathrm{Mg}+$ $0.9 \mathrm{~K} * \mathrm{Zn}+0.02 \mathrm{Mg} * \mathrm{Zn}$

The resolution of this system of equations obtained through the partial deriv- 
atives of the equation to the marginal product lead to the following optimal doses of each nutrient: $16.01 \mathrm{~kg} \mathrm{~N} \mathrm{ha}^{-1}, 20.18 \mathrm{~kg} \mathrm{P} \mathrm{ha}^{-1}, 6.70 \mathrm{~kg} \mathrm{~K} \mathrm{ha}^{-1}, 5.65 \mathrm{Kg} \mathrm{Mg}$ $\mathrm{ha}^{-1}$ and $2.47 \mathrm{Kg} \mathrm{Zn}$ at the site of Centre Benin and $13.10 \mathrm{~kg} \mathrm{~N} \mathrm{ha}^{-1}, 25.07 \mathrm{~kg} \mathrm{P}$ $\mathrm{ha}^{-1}, 11.47 \mathrm{~kg} \mathrm{Mg} \mathrm{ha}^{-1}, 0 \mathrm{~kg} \mathrm{~K} \mathrm{ha}^{-1}$ and $1.82 \mathrm{~kg} \mathrm{Zn} \mathrm{ha}^{-1}$ at the site of North Benin. These optimal doses have induced an optimal groundnut pod yield of $2.1 \mathrm{t} \cdot \mathrm{ha}^{-1}$.

\section{Discussions}

\subsection{Groundnut Response to Mineral Fertilizer}

Application of nutrients significantly improved groundnut growth parameters especially root length. This root elongation enables good soil depth exploration for nutrients uptake. The increase in root length was due to the supply of $\mathrm{P}$ which is known to develop more extensive plant rooting system [27] and probably to $\mathrm{Mg}$ and $\mathrm{Zn}$. Indeed, $\mathrm{Mg}$ and $\mathrm{Zn}$ contribute to the solubilization of insoluble phosphorus in the soil and also contribute to improve $\mathrm{P}$ absorption.

In addition, groundnut recovery diameters were significantly affected by the types and rates of nutrient applied. This is an indicator predicting plant development and the aboveground biomass production [25]. Our result showed that different rates of $\mathrm{P}$ and $\mathrm{Zn}$ induced significant effects on groundnut recovery diameter. This could be explained by the important role of these nutrients in cell division, which actively participates in the rapid development of meristematic tissues resulting in a large number of leaves and plant height growth [28] [29] [30]. This high plant recovery diameter actively participates in the photosynthesis and plant aboveground biomass production.

However, more gynophores and number of nodules were recorded during the present experiment. The high number of nodules per plant could be explained by the presence of $\mathrm{P}$ in the nutrient applied which is important in the establishment of nodules in legumes [31]. However, $\mathrm{P}$ ensures energy transfer, a cellular constituent that ensures the synthesis of nucleic acids and increases the number of groundnut nodules per plant. This had increased probably the atmospheric nitrogen fixation [32]. The Gynophores are an essential component of groundnut yield as they are directly related to the number of pods production. The results of the present study showed that treatments with intermediate doses of $\mathrm{P}, \mathrm{K}$ and $\mathrm{Mg}$ produced high number of gynophores. It is well known that $\mathrm{N}$ is involved in photosynthesis, and ensures also a good development of the gynophores. $\mathrm{P}$ interacts with $\mathrm{N}$ through its ability to provide energy to the photosynthesis process and $\mathrm{N}$ biosynthesis, which activates the production of gynophores. [33] reported that N, P and $\mathrm{K}$ doses significantly increase the number of gynophores in groundnut crops. In general, based on the results of this study, the nutrients applied contributed to the growth and development of groundnut through their role in the plant. This may have contributed to pod yields improvement.

Compared to the control, the different types and rates of nutrient applied have increased by 2.5 times the pod yields. The positive effects of nutrients on legume 
pod yields were observed in several studies [34] [35]. The increase in the pod yields was probably due to the improvement of plant nutritional status. Furthermore, interaction of $\mathrm{Mg}$ and $\mathrm{Zn}$ with $\mathrm{N}, \mathrm{P}$ and $\mathrm{K}$ have highly influenced groundnut yield. The highest pod yields were registered with $15 \mathrm{~kg} \mathrm{Mg} \mathrm{ha}^{-1}$ and $4 \mathrm{~kg} \mathrm{Zn} \mathrm{ha}^{-1}$ combined with intermediate doses of $\mathrm{N}, \mathrm{P}$ and $\mathrm{K}$. These high pod yields could be explained by the fact that the absorption of $\mathrm{Mg}$ and $\mathrm{Zn}$ improved the efficiency of macronutrient $(\mathrm{N}, \mathrm{P}$, and $\mathrm{K})$ utilization by groundnut plants [16]. Indeed, $\mathrm{Mg}$ promotes the assimilation and migration of $\mathrm{P}$ in the plant and in the seeds to form phytine and lipids [21]. It is therefore suggested to optimize plant mineral nutrition to ensure yield improvement in the traditional cropping system.

\subsection{Relevance of Mg and Zn in Groundnut Mineral Nutrition Improvement}

Groundnut pod yields and aboveground biomass were significantly improved regarding the types and rates of nutrient applied combined with $\mathrm{Zn}$. Our results also showed that pod yields were influenced by nutrient rates and interaction of $\mathrm{N}, \mathrm{P}$ and $\mathrm{K}$ with $\mathrm{Mg}$ and $\mathrm{Zn}$. The $\mathrm{N}, \mathrm{P}$ and $\mathrm{K}$ use efficiencies were thus improved due to the uptake of $\mathrm{Mg}$ and $\mathrm{Zn}$. These results corroborated that of [36] who showed that micronutrients significantly increase crop yields and were the main pillars for soil fertility management. Indeed, macronutrients are better taken up by the crops in the presence of micronutrients [20]. However, Mg promotes the uptake and migration of $\mathrm{P}$ in the plant and seeds and form phytine and lipids [21]. The levels of micronutrients in the arable soils in most of the sub-Saharan Africa's soil are below the critical threshold, particularly $\mathrm{Zn}$ [37]. The combined application of macronutrients with $\mathrm{Mg}$ and $\mathrm{Zn}$ has induced significant effect on macronutrients utilization efficiency. [15] showed that the addition of micronutrients with $\mathrm{N}, \mathrm{P}$ and $\mathrm{K}$ resulted in yield improvement by $50 \%$ compared with application of N, P and S without micronutrients. [38] also showed that $\mathrm{Zn}$ application had increase macronutrients uptake in the plant and improved $\mathrm{N}, \mathrm{P}$ and $\mathrm{K}$ use efficiency. Supply of $\mathrm{Zn}$ has induced $\mathrm{N}$ use efficiency by $17 \%$ leading to crop productivity improvement. When micronutrients were applied at appropriate rate, it is also beneficial to the soil biodiversity, including soil microbial colonization, plant growth, mycorrhizal development, legume fixation and nodulation [39]. Our study suggests supply of $\mathrm{Zn}$ and $\mathrm{Mg}$ to improve groundnut nutritional status in the farmers' cropping systems for pod yields improvement and for sustainable soil fertility management.

\subsection{Agronomic Implication of the Optimal N, P, K, Mg and Zn Rates Determined}

The application of the different nutrient $\mathrm{N}, \mathrm{P}, \mathrm{K}, \mathrm{Mg}$ and $\mathrm{Zn}$ and rates showed a normal crop response except $\mathrm{K}$ for which no response was observed in the site of the north. The optimal dose of $0 \mathrm{~kg} \mathrm{~K} \mathrm{ha}^{-1}$ found for this site means that no external $\mathrm{K}$ input is required to achieve optimal pod yield. This may be due to the 
fact that the reserve of potassium in these soils was sufficient to ensure or meet $\mathrm{K}$ needs for groundnut crop. Similarly, optimization of crop nutrition should lead to the balanced and efficient nutrient use and also to minimize adverse effect on the environment [40]. This can be achieved by combining the optimal nutrient inputs with best crop management practices, hence the law of minimum. However, the optimal dose of $0 \mathrm{~kg} \mathrm{~K} \mathrm{ha}^{-1}$ cannot be recommended for farmers regarding the sustainability of the cropping system [41]. We recommend the minimum dose of $20 \mathrm{~kg} \mathrm{~K} \mathrm{ha}^{-1}$ in order to guarantee sustainable soil production.

According to [42] the doses of $50 \mathrm{~kg} \cdot \mathrm{ha}^{-1}$ of $\mathrm{P}_{2} \mathrm{O}_{5}$ to $75 \mathrm{~kg} \cdot \mathrm{ha}^{-1}$ are sufficient to improve seed oil content in addition to the pod yields. The different optimal doses found in the present study could then allow seeds with high oil content though this needs to be tested. Also, these N, P, K, Mg and Zn doses determined can guarantee profitable economic production as well as good quality product and by-product (oil, cake, groundnut paste) and finally quality fodder for animal feed. The next step of the study is to formulate an N-P-K-Mg-Zn fertilizer for farmers and to assess the climate variability effect on the yields regarding the doses determined.

\section{Conclusion}

Mineral fertilization of groundnut with $\mathrm{N}, \mathrm{P}, \mathrm{K}, \mathrm{Mg}$ and $\mathrm{Zn}$ improved the agronomic parameters of groundnut showing a positive response of groundnut to mineral fertilization. Our results indicated that the application of $\mathrm{N}, \mathrm{P}$ and $\mathrm{K}$ combined with $\mathrm{Zn}$ improves nodule production and groundnut growth parameters. The pod yields and aboveground biomass were significantly improved regarding the treatments. This is relevant that legume crops in the farmers' cropping system in Benin need appropriate fertilizer formulae to improve plant nutritional status. Response surface analysis showed doses of each nutrient: 16.01 $\mathrm{kg} \mathrm{N} \mathrm{ha}{ }^{-1}, 20.18 \mathrm{~kg} \mathrm{P} \mathrm{ha}^{-1}, 6.70 \mathrm{~kg} \mathrm{~K} \mathrm{ha}^{-1}, 5.65 \mathrm{Kg} \mathrm{Mg} \mathrm{ha}^{-1}$ and $2.47 \mathrm{Kg} \mathrm{Zn} \mathrm{for}$ the site of Centre Benin and $13.10 \mathrm{~kg} \mathrm{~N} \mathrm{ha}^{-1}, 25.07 \mathrm{~kg} \mathrm{P} \mathrm{ha}^{-1}, 11.47 \mathrm{~kg} \mathrm{Mg} \mathrm{ha}^{-1}, 0$ $\mathrm{kg} \mathrm{K} \mathrm{ha} a^{-1}$ and $1.82 \mathrm{~kg} \mathrm{Zn} \mathrm{ha}^{-1}$ for the site of North Benin for sustainable groundnut production. These optimal doses have induced an optimal groundnut pod yields of $2.1 \mathrm{t} \cdot \mathrm{ha}^{-1}$. In order to avoid $\mathrm{K}$ mining in the soils as suggested by the model, a minimal dose of $20 \mathrm{~kg} \mathrm{~K} \mathrm{ha}^{-1}$ was suggested. For sustainable groundnut production perspective, it would be interesting to evaluate the effect of long-term climate variability on the pods' yields considering the determined optimal N, P, K, Mg and Zn doses. Fertilizer formulations based on these optimal N, P, K, Mg and $\mathrm{Zn}$ doses are the next step of the programme in order to make available specific fertilizer for groundnut production in Benin.

\section{Acknowledgements}

The authors thank the Improvement of Smallholder Agricultural Productivity (PAPAPE/SAPEP-Benin) of Benin National Agricultural Research Institute (INRAB), funded by the Islamic Development Bank (IDB) and Benin Govern- 
ment, for the financial support of the present research program.

\section{Conflicts of Interest}

The authors declare no conflicts of interest regarding the publication of this paper.

\section{References}

[1] Aher, S.B., Lakaria, B.L., Kaleshananda, S., Singh, A.L., Ramana, S., Ramesh, K. and Thakur, J.K. (2015) Effect of Organic Farming Practices on Soil and Performance of Soybean (Glycine max) under Semi-Arid Tropical Conditions in Central India. Journal of Applied and Natural Science, 94, 67-71. https://doi.org/10.31018/jans.v7i1.564

[2] Sultana, R., Vales, M.I., Saxena, K.B., Rathore, A., Rao, S., Rao, S.K., Mula, M.G. and Kumar R.V. (2014) Waterlogging Tolerance in Pigeonpea (Cajanus cajan (L.) Millsp.): Genotypic Variability and Identification of Tolerant Genotypes. The Journal of Agricultural Science, 151, 659-671. https://doi.org/10.1017/S0021859612000755

[3] Karaboneye, F. (2013) Caractérisation de l'efficacité symbiotique de lignées africaines de soya à haute promiscuité. Mémoire Master, Université Lawal.

[4] Zeinabou, H., Mahamane, S., Bismarck, N.H., Bado, B. V., Lompo, F. and Bationo, A. (2014) Effet de la combinaison des fumures organo-minérales et de la rotation niébé-mil sur la nutrition azotée et les rendements du miel au sahel. International Journal of Biological and Chemical Sciences, 8, 1620-1632.

https://doi.org/10.4314/ijbcs.v8i4.24

[5] Bado, B.V. (2018) Rôle des légumineuses sur la fertilité des sols ferrugineux tropicaux des zones guinéenne et soudanienne du Burkina Faso. PhD, Université Laval, Canada.

[6] Gogoi, N., Baruah, K.K. and Meena, R.S. (2018) Grain Legumes: Impact on Soil Health and Agroecosystem. In: Meena, R.S., Das, A., Yadav, G.S. and Lal, R., Eds., Legumes for Soil Health and Sustainable Management, Springer, Singapore, 511-539. https://doi.org/10.1007/978-981-13-0253-4_16

[7] Ghosh, P., Bandyopadhyay, K., Wanjari, R., Manna, M., Misra, A., Mohanty, M. and Rao, A.S. (2007) Legume Effect for Enhancing Productivity and Nutrient Use-Efficiency in Major Cropping Systems-An Indian Perspective: A Review. Journal of Sustainable Agriculture, 30, 59-86. https://doi.org/10.1300/J064v30n01_07

[8] Dugje, I.Y., Omoigui, L.O., Ekeleme, F., Kamara, A.Y. and Ajeigbe, H. (2009) Farmers' Guide to Cowpea Production in West Africa. IITA, Ibadan, 20, 12-14.

[9] Leghari, S.J., Wahocho, N.A., Laghari, G.M., HafeezLaghari, A., MustafaBhabhan, G., HussainTalpur, K. and Lashari, A.A. (2016) Role of Nitrogen for Plant Growth and Development: A Review. Advances in Environmental Biology, 10, 209-219.

[10] Afolabi, S.G., Adekanmbi, A.A., Adeboye, M.K. and Bala, A. (2014) Effect of Various Input Combinations on the Growth, Nodulation and Yield of Inoculated Soybean in Minna. Nigeria International Journal of Agricultural and Rural Development, 17, 2006-2011.

[11] Vanlauwe, B. and Giller, K.E. (2006) Popular Myths around Soil Fertility Management in Sub-Saharan Africa. Agriculture, Ecosystems and Environment, 116, 34-46. https://doi.org/10.1016/j.agee.2006.03.016 
[12] Kihara, J., Sileshi, G.W., Nziguheba, G., Kinyua, M., Zingore, S. and Sommer, R. (2017) Application of Secondary Nutrients and Micronutrients Increases Crop Yields in Sub-Saharan Africa. Agronomy for Sustainable Development, 37, 25. https://doi.org/10.1007/s13593-017-0431-0

[13] Chianu, J.N. and Mairura, F. (2012) Mineral Fertilizers in the Farming Systems of Sub-Saharan Africa. A Review. Agronomy for Sustainable Development, 32, 545-566. https://doi.org/10.1007/s13593-011-0050-0

[14] Vanlauwe, B., Descheemaeker, K., Giller, K.E., Huising, J., Merckx, R., Nziguheba, G., Wendt, J. and Zingore, S. (2015) Integrated Soil Fertility Management in Sub-Saharan Africa: Unravelling Local Adaptation. Soil, 1, 491-508. https://doi.org/10.5194/soil-1-491-2015

[15] Kihara, J., Bolo, P., Kinyua, M., Rurinda, J. and Piikki, K. (2020) Micronutrient Deficiencies in African Soils and the Human Nutritional Nexus: Opportunities with Staple Crops. Environmental Geochemistry and Health, 1-19. https://doi.org/10.1007/s10653-019-00499-w

[16] Yosefi, K., Galavi, M., Ramrodi, M. and Mousavi, S.R. (2011) Effect of Bio-Phosphate and Chemical Phosphorus Fertilizer Accompanied with Micronutrient Foliar Application on Growth, Yield and Yield Components of Maize (Single Cross 704). Australian Journal of Crop Science, 5, 175-180.

[17] Prasad, T.N.V.K.V., Sudhakar, P., Sreenivasulu, Y., Latha, P., Munaswamy, V., Reddy, K.R., Sreeprasad, T.S., Sajanlal, P.R. and Pradeep, T. (2012) Effect of Nanoscale Zinc Oxide Particles on the Germination, Growth and Yield of Peanut. Journal of Plant Nutrition, 35, 905-927. https://doi.org/10.1080/01904167.2012.663443

[18] Wakeel, A., Farooq, M., Bashir, K. and Ozturk, L. (2018) Micronutrient Malnutrition and Biofortification: Recent Advances and Future Perspectives. In: Plant Micronutrient Use Efficiency, Academic Press, Cambridge, 225-243. https://doi.org/10.1016/B978-0-12-812104-7.00017-4

[19] Malekzadeh-Shafarod, S., Bidarigh, S. and Sadeghi, S.M. (2013) The Evaluation on Effect of Nitrogen Fertilizer and Chelated Zinc on the Yield of Peanut (Arachis hypogae). Indian Journal of Fundamental and Applied Life Sciences, 3, 95-103.

[20] Yu-Chuan, N.D.D. (2011) Effects of Different Magnesium Fertilizers on Growth and Nutrient Uptake of Soybean Plants. Modern Agricultural Sciences and Technology, No. 6, 188.

[21] Walker, M.E., Gaines, T.P. and Parker, M.B. (1989) Potassium, Magnesium, and Irrigation Effects on Peanuts Grown on Two Soils. Communications in Soil Science and Plant Analysis, 20, 1011-1032. https://doi.org/10.1080/00103628909368132

[22] Li, Y., Li, Q.H., Wang, F., He, C.M. and Lin, X.J. (2007) Effects on Yield Quality and Benefit of Applying Homemade Sulphate-Potassium Magnesium Fertilizer to Peanut. Journal of Peanut Science, 1, 1-10.

[23] Chabi, F.O., Dagbenonbakin, G.D., Oussou, B.T. and Saïdou, A. (2019) Determinant of Groundnut (Arachis hypogaea L.) Yield Improvement in the Farmers Cropping Systems in Benin. African Journal of Agricultural Research, 14, 1967-1979.

[24] FAO (2015) World Reference Base for Soil Resources 2014, Update 2015 International Soil Classification System for Naming Soils and Creating Legends for Soil Maps. World Soil Resources Reports No. 106. FAO, Rome.

[25] Bloukounon, A.Y.G., Saïdou, A., Babatounde, S., Balogoun, I., Arakogne, S., Kassavi, E. and Adegbidi, A. (2015) Effets des fumures NPK et déjections de petits ruminants sur la productivité et la valeur fourragère du maïs et de l'arachide au Sud-Bénin. Annales des Sciences Agronomiques, 19, 213-238.

[26] Yolmeh, M. and Jafari, S.M. (2017) Applications of Response Surface Methodology 
in the Food Industry Processes. Food and Bioprocess Technology, 10, 413-433. https://doi.org/10.1007/s11947-016-1855-2

[27] Gobarah, M.E., Mohammad, M.H. and Tawfik, M.M. (2006) Effect of Phosphorus Fertilizer and Foliar Spraying with Zinc on Growth, Yield and Quality of Groundnut under Reclaimed Sandy Soils. Journal of Applied Science Research, 2, 491-496.

[28] Hemalatha, S., Praveen Rao, V., Padmaja, J. and Suresh, K. (2013) Anoverview on Role of Phosphorus and Water Deficits on Growth, Yield and Quality of Groundnut (Arachis hypogaea L.). International Journal of Applied Biology and Pharmaceutical Technology, 4, 188-201.

[29] Farag, I.A.A. and Ahmed, A.Z. (2014) Croissance de l'arachide (Arachis hypogaea L.) et réponses de rendement à l'irradiation des graines et à la fertilisation minérale. IOSR Journal of Agriculture and Veterinary Science, 7, 63-70. https://doi.org/10.9790/2380-07536370

[30] Silva, E.D.B., Ferreira, E.A., Pereira, G.A.M., Silva, D.V. and Oliveira, A.J.M. (2017) Peanut Plant Nutrient Absorption and Growth. Revista Caatinga, 30, 653-661. https://doi.org/10.1590/1983-21252017v30n313rc

[31] Sulieman, S. and Tran, L.S.P. (2015) Phosphorus Homeostasis in Legume Nodules as an Adaptive Strategy to Phosphorus Deficiency. Plant Science, 239, 36-43. https://doi.org/10.1016/j.plantsci.2015.06.018

[32] Yusif, S.A., Muhammad, I., Hayatu, N.G., Sauwa, M.M., Tafinta, I.Y., Mohammed, M.A., Lukman, S.A., Abubakar, G.A. and Hussain, A.M. (2016) Effects of Biochar and Rhizobium Inoculation on Nodulation and Growth of Groundnut in Sokoto State, Nigeria. Journal of Applied Life Sciences International, 9, 1-9. https://doi.org/10.9734/JALSI/2016/27297

[33] Hadwani, G.J. and Gundalia, J.D. (2005) Effect of N, P and K Levels on Yield, Nutrient Content, Uptake and Quality of Summer Groundnut Grown on Typic Haplustepts. Journal of the Indian Society of Soil Science, 53, 125-128.

[34] Irmak, S., Cil, A., Yucel, H. and Kaya, Z. (2015) Effects of Zinc Application on Yield and Some Yield Components in Peanut (Arachis hypogaea) in the Easthern Mediterranean Region. Journal of Agricultural Science, 22, 109-116. https://doi.org/10.1501/Tarimbil_0000001373

[35] Sengupta, A., Gunri, S.K. and Basu, T.K. (2016) Performance of Short Duration Groundnut (Arachis hypogaea L.) Variety (TG 51) as Influenced by Nutrient Management Strategy under New Alluvial Zone of West Bengal. Legume Research: An International Journal, 39, 91-95. https://doi.org/10.18805/lr.v39i1.8869

[36] Tavakoli, M.T., Chenari, A.I., Rezaie, M., Tavakoli, A., Shahsavari, M. and Mousavi, S.R. (2014) The Importance of Micronutrients in Agricultural Production. Advances in Environmental Biology, 31-36.

[37] Cakmak, I., McLaughlin, M.J. and White, P. (2017) Zinc for Better Crop Production and Human Health. Plant and Soil, 4, 1-4.

https://doi.org/10.1007/s11104-016-3166-9

[38] El-Dahshouri, M.F. (2018) Effect of Zinc Foliar Application at Different Physiological Growth Stages on Yield and Quality of Wheat under Sandy Soil Conditions. Agricultural Engineering International: CIGR Journal, 19, 193-200.

[39] Farooq, M., Ullaha, A., Rehmana, A., Nawaza, A., Nadeema, A., Wakeele, A., Nadeema, F. and Siddiquec, K.H.M. (2018) Application of Zinc Improves the Productivity and Biofortification of Fine Grain Aromatic Rice Grown in Dry Seeded and Puddled Transplanted Production Systems. Field Crops Research, 216, 53-62. https://doi.org/10.1016/j.fcr.2017.11.004 
[40] Singh, A.L. and Chaudhari, V. (2015) Zinc Biofortification in Sixty Groundnut Cultivars through Foliar Application of Zinc Sulphate. Journal of Plant Nutrition, 38, 1734-1753. https://doi.org/10.1080/01904167.2015.1042165

[41] Saïdou, A., Balogoun, I., Ahoton, E.L., Igué, A.M., Youl, S. and Ezui, G. (2017) Fertilizer Recommendations for Corn Production in the South Sudan and Sudano-Guinean Zones of Benin. Nutrient Cycling in Agroecosystems, 110, 361-373. https://doi.org/10.1007/s10705-017-9902-6

[42] Abbas, Z., Kumar, A. and Kumar, A. (2018) Peanut Agriculture and Production Technology Integrated Nutrient Management. Apple Academic Press Inc., Oakville. https://doi.org/10.1201/9781315166872 